\title{
Synthesis and Pharmacological Evaluation of Newer Substituted 2-oxo/thiobarbiturinylbenzoxa/thiazepine Derivatives as Potent Anticonvulsant Agents
}

\author{
MIRDULATYAGI* and ARCHANA \\ Department of Chemistry, Medicinal Chenmistry Lab Meerut College, Meerut-250004 (U.P.) India. \\ ${ }^{*}$ Corresponding author E-mail: mirdulatyagi@gmail.com \\ http://dx.doi.org/10.13005/ojc/310113
}

(Received: December 25, 2014; Accepted: January 20, 2015)

\begin{abstract}
A new series of 4-(2'-Oxo/thiobarbiturinyl)-2-(substitutedphenyl)-3-[(substituted aminomethylene)]-2,3-dihydro-1,5-benzothiazepines (5a-5I) and 4-(2'-oxo/thiobarbiturinyl)-2(substituted-phenyl)-3-(substitutedaminomethylene)]-2,3-dihydro-1, 5- benzoxazepines (6a-6l) were synthesized. All the newly synthesized compounds were screened in vivo, for their acute toxicity and anticonvulsant activity in MES and PTZ models and were compared with standard drugs phenytoin sodium and sodium valporate. Out of the compounds studied, the most active compound of this series was 5 h, showed activity (90\%) more potent than the standard drug.
\end{abstract}

Key words: Anticonvulsant Agents, in vivo, standard drugs.

\section{INTRODUCTION}

The barbiturates comprise an important and valuable class of central nervous system depressants. Barbituric acid (2,4,6trioxohexahydrophrimidine) derivative, phenobarbital and mephobarbital are used for clinical treatment of epilepsy. Further substitution pattern at $5^{\text {th }}$ position of barbituric acid ${ }^{3-8}$ by different alkyl, aryl or heteroaryl moieties plays a pivotal role in modulation of anticonvulsant activity. Moreover, compounds containing a fused seven, membered heterocyclic ring i.e. bengzothiazepine/ benzoxazepine nucleus make up a broad class that attracted attention in the past few years owing to its wide range of biological activities especially anticonvulsant and CNS depressant activities ${ }^{9-14}$. However, these compounds have not been in clinical use as they possess either less activity or more side effects.

Incorporating these moieties in $5^{\text {th }}$ position of 2-oxo/thiobarbituric acid nucleus might be thought to yield more potent anticonvulsant compounds as substituted moieties are themselves anticonvulsant and substituting at $5^{\text {th }}$ position further results in protecting against convulsions. Thus, the substitution by these moieties may be synergistic. The present project is therefore, aimed at synthesizing such compounds. 


\section{Chemistry}

The synthetic routes of compounds are outlined in scheme 1. 5-Acetyl-2-oxo/thiobarbituric acid $1 \mathrm{a}-1 \mathrm{~b}$ weresynthesized by the reaction of 2 oxo/thiobarbituric acid and acetyl chloride. Compounds $1 \mathrm{a}-1 \mathrm{~b}$ on reaction with different aromatic aldehydes yielded 1-(2'-oxo/ thiobarbiturinyl)-3-chalcones i.e. compounds 2a2fwhich on cyclization with 2-thio/aminophenol in presence of glacial acetic acid yielded compounds $3 a-3 f$ and $4 a-4 f$ respectively. compounds $3 a-3 f$ and $4 \mathrm{a}-4 \mathrm{f}$ further undergoes Mannichreaction with different substituted anilines to afford compounds $5 a-51$ and $6 a-6 l$.

\section{RESULTS AND DISCUSSION}

Anticonvulsant activity and acute toxicity of these new substituted 2-oxo/ thiobarbiturinylenzoxa/thiazepine derivatives 2a-2f, $3 a-3 f, 4 a-4 f, 5 a-5 l$ and $6 a-6 l$ are represented in Table-1.

Anticonvulsant activity (maximum electroshock induced seizures and pentylenetetrazol induced seizure pattern test)

The characteristic feature of the compounds of this series is the incorporation of two heterocyclic moieties, that is 2-oxo/thiobarbituric acid and benzoxazepine/benzothiazepine into a single molecular framework with the aim to develop more potent anticonvulsant agents withminimum or no side effects.

5-Acetyl-2-oxo/thiobarbituric acids $1 \mathrm{a}-1 \mathrm{~b}$ showed $10-20 \%$ anticonvulsant activity at a dose of $50 \mathrm{mg} / \mathrm{kg}$ i.p. in maximal electroshock and pentylenetetrazole induced seizures, respectively. Screening of step-2 compounds 1-(2'-oxo/ thiobarbiturinyl)-3-arylidenylchalcones $2 a-2 f$ revealed that these compoundsshowed somewhat increase in anticonvulsant activity in both the models (ranging from 20 to $50 \%$ and 10 to $50 \%$ in MES and PTZ models, respectively) in comparison to step-1 compounds when tested at same dose.

Compounds 4-(2'-oxo/thiobarbiturinyl)-2substitutedphenyl)-2,3,-dihydro-1,5benzothiazepines $3 a-3 f$ showed high percentage protection ranging from 40 to $80 \%$ and 30 to $80 \%$ in
MES and PTZ models, respectively. The most active compound among $3 \mathrm{a}-3 \mathrm{f}$ is compound $3 \mathrm{~d}$. This compound was found to be equipotent $(80 \%$ protection) to phenytoin sodium (standard drug for MES model) and sodium valproate (standard drug for PTZ model) and hence due to its potent nature it was studied in detail at three graded doses (17.5, 25 and $50 \mathrm{mg} / \mathrm{kg}$ i.p.) for its anticonvulsant activity and was found to possess $20,40,80 \%$ and 20,30 , $80 \%$ protection of seizures in MES and PTZ models, respectively. It was also observed that compound 3c (having 3-methoxyphenyl group) showed least activity $40 \%$ while compound $3 d$ (having 2 chlorophenyl group) exhibited maximum response $80 \%$ in comparison to other substituted compounds. Further compounds having thiobarbituric acid possess more potent activity than the compounds having oxobarbituricacid ring.

Further, the next step compounds(5a-5l) was characterized by presence of different arylaminomethylene substitutions at the third position of benzothiazepine ring. They exhibited potent anticonvulsant activity ranging from 60 to $90 \%$ and 50 to $90 \%$ inbothmodels, that is MES and PTZ respectively. Out of the twelve compounds 5a$5 \mathrm{l}$ the most active compound is $5 \mathrm{~h}$. (having 4methoxyphenyl aminomethylen substitution at third position of benzothiazepine ring and 2chlorophenyl substitution at second position of benzodiazepine ring) was found to be most potent compound of this series exhibiting $90 \%$ inhibition in both MES and PTZ models. This compound was found to be more potent ( $90 \%$ protection) to phenytoin sodium (standard drug for MES model) and sodium valproate (standard drug for PTZ model). This compound was studied in details at three graded doses $(17.5,25$ and $50 \mathrm{mg} / \mathrm{kg}$ i.p.) for its anticonvulsant activity and was found to possess $20,50,90 \%$ and $20,40,90 \%$ protection of seizures in MES and PTZ models, respectively. On the other hand compounds $4 a-4 f$ possessed benzoxazepine ring with 2-oxo/thiobarbituric acid, substituted by different moieties at second position exhibited aniconvulsnat activity ranging from 40 to $70 \%$ and 30 to $60 \%$ in both MES and PTZ models respectively. So the compounds $4 a-4 f$ showed a decrease in anticonvulsant activity in comparison to compounds 3a-3f i.e. benzothiazepine compounds.Further the compounds of next step i.e. 6a-6l were characterized 
by different arylaminomethylene substitutions at the third position of benzoxazepine ring. All the twelve compounds $6 a-6 \mathrm{l}$ of this step exhibited anticonvulsant activity ranging from 50 to $70 \%$ and 40 to $60 \%$ protection of seizures in MES and PTZ models, respectively.

\section{ALD $_{50}$ Studies}

The toxicity study of these compounds indicate their good safety margin.

\section{EXPERIMENTAL}

\section{Chemistry}

Melting points were determined in open capillaries with the help of thermonic melting point apparatus and are uncorrected. 1R spectra $(\mathrm{KBr})$ are recorded on Backmann Acculab-10 spectrophotometer. ${ }^{1} \mathrm{H}$ NMR spectra were recorded by Bruker WM $400 \mathrm{FT}$ instrument using $\mathrm{CDCl}_{3}$ as solvent and tetramethylsilane (TMS) as internal reference standard. All chemical shift (d)are in ppm. The purities of the compounds were checked by thin layer chromatography (TLC) on silicagel-G plates of $0.5 \mathrm{~mm}$ thickness. The elemental analysis of the compounds were performed on HeracusCarlo Erba 1108 analyser.

5-Acetyl-2-oxobarbituric acid 1a. Acetyl chloride $(50 \mathrm{ml})$ was added to 2-oxobarbituric acid $(20 \mathrm{~g})$ drop by drop with stirring at $0-5^{\circ} \mathrm{C}$. The reaction mixture was further stirred for $10 \mathrm{~h}$ using a magnetic stirrer and kept overnight. The excess of acetyl chloride was distilled off with the help of a distillation assembly and the residue thus obtained was washed with petroleum ether $40-60^{\circ} \mathrm{C}$, a number of times and then poured onto ice. The solid thus obtained was filtered with the help of a filteration pump and recrystallised from methanol/water to give compounds $1 \mathrm{a}(75 \%), \mathrm{mp} 180^{\circ} \mathrm{C}$; $1 \mathrm{R}(\mathrm{KBr})$ $3100(\mathrm{NH}), 1750,1720,1700,1690,(\mathrm{C}=\mathrm{O}) \mathrm{cm}^{-1}$; ${ }^{1} \mathrm{HNMR}\left(\mathrm{CDCl}_{3}\right)$ of 9.25 (ss, 2H, 2NHCO), 5.79 (s, $\left.1 \mathrm{H}, \mathrm{CHCOCH}_{3}\right), 2.47$ (s, 3H, $\left.\mathrm{COCH}_{3}\right)(\mathrm{ppm}) ; \mathrm{MS}$ : $\mathrm{M}^{+}$170. Anal.calcd. for $\mathrm{C}_{6} \mathrm{H}_{6} \mathrm{~N}_{2} \mathrm{O}_{4} ; \mathrm{C}, 42.35 ; \mathrm{H}, 3.52$; N, 16.47. Found: C, 42.33; H, 3.50; N, 16.51.

-Acetyl-2-thiobarbituric acid (1b). 77\%,m.p. $250^{\circ} \mathrm{C}$ (methanol/water); 1R ( $\left.\mathrm{KBr}\right) 3140(\mathrm{NH}), 1133$ $(\mathrm{C}=\mathrm{S}) \mathrm{cm}^{-1} ; 1 \mathrm{H} \mathrm{NMR}\left(\mathrm{CDCl}_{3}\right)$ d 9.28 (ss, 2H, 2NHCO), $5.71\left(\mathrm{~s}, 1 \mathrm{H}, \mathrm{CHCOCH}_{3}\right), 2.50\left(\mathrm{~s}, 3 \mathrm{H}, \mathrm{COCH}_{3}\right)(\mathrm{ppm})$;
MS : $\mathrm{M}^{+}$178. Anal. calcd. for $\mathrm{C}_{6} \mathrm{H}_{6} \mathrm{~N}_{2} \mathrm{O}_{3} \mathrm{~S} ; \mathrm{C}, 38.70$; $\mathrm{H}, 3.22 ; \mathrm{N}, 15.05$. Found :C, 38.71; H, 3.20; N, 15.07 .

1-(21-Oxobarbiturinyl)-3-(2-chlorophenyl) chalcone (2a). A mixture of 5-acetyl-2-barbituric acid (0.01 mole) and 2-chloroaldehyde (0.01 mole) in absolute methanol $(50 \mathrm{ml})$ in presence of $2 \%$ $\mathrm{NaOH}$ were refluxed for $12 \mathrm{~h}$. The resulting mixture was concentrated, cooled and poured onto ice. The solid thus obtained was filtered, washed with petroleum ether $\left(40-60^{\circ} \mathrm{C}\right)$ and recrystallised from methanol/water to give compound $2 \mathrm{a}(90 \%), \mathrm{mp}$ $240^{\circ} \mathrm{C}$; IR $(\mathrm{KBr}) 3320(\mathrm{NH}), 1620(\mathrm{CH}=\mathrm{CH}), 1730$, 1720, 1715, 1680 (amidic $(\mathrm{C}=\mathrm{O}), 1580$ (C...C of aromatic ring), $710(\mathrm{C}-\mathrm{Cl}) \mathrm{cm}^{-1} ;{ }^{1} \mathrm{H}$ NMR $\left(\mathrm{CDCl}_{3}\right) \mathrm{d}$ $5.80(\mathrm{~m}, 1 \mathrm{H}, \mathrm{CHCO}), 6.65(\mathrm{~d}, 1 \mathrm{H},-\mathrm{COCH}=), 7.80$ 7.27 (m, 4H,Ar-H), 8.30 (d, 1H, = CH-Ar), 9.30 (ss, 2H, 2NHCO) (ppm); MS : $\mathrm{M}^{+}$292.5. Anal.calcdfor $\mathrm{C}_{13} \mathrm{H}_{9} \mathrm{~N}_{2} \mathrm{O}_{4} \mathrm{Cl} ; \mathrm{C}, 53.33 ; \mathrm{H}, 3.07 ; \mathrm{N}, 9.57$. Found C,53.30; H, 3.02; N, 9.59 .

1-(2'-Oxobarbituriyl)-3-(p-hydroxyphenyl)chalcone (2b). 85\%, mp 250ㄷ (DMF); $1 \mathrm{R}(\mathrm{KBr})$ $3320(\mathrm{NH}), 1620(\mathrm{CH}=\mathrm{CH}), 1690,1710,1715$ (amidic $\mathrm{C}=\mathrm{O}), 1530\left(\mathrm{C}_{\ldots} . \mathrm{C}\right)$ of aromatic ring $\mathrm{cm}^{-1}$; ${ }^{1} \mathrm{H} \mathrm{NMR}\left(\mathrm{CDCl}_{3}\right)$ d $9.10(3,1 \mathrm{H}, \mathrm{Ar}-\mathrm{OH}) 5.80(\mathrm{~m}, 1 \mathrm{H}$, $\mathrm{CHCO}), 6.65(\mathrm{~d}, 1 \mathrm{H},-\mathrm{COCH}=), 7.10-8.10(\mathrm{~m}, 4 \mathrm{H}$, $\mathrm{Ar}-\mathrm{H}$ ), 8.48 (d,1H, =CH-Ar), 9.25 (ss, 2H, 2NHCO) (ppm); MS : $\mathrm{M}^{+}$258. Anal.Calcd.for $\mathrm{C}_{13} \mathrm{H}_{10} \mathrm{~N}_{2} \mathrm{O}_{5} ; \mathrm{C}$, 60.46 ; H, 3.93 ; N, 10.85. Found : C. $60.40 ; \mathrm{H}, 3.85$; $\mathrm{N}, 10.88$

1-(2'-Oxobarbiturinyl) -3-(mmethoxyphenyl)-chalcone (2c). $80 \%, \mathrm{mp} 280^{\circ} \mathrm{C}$ (methanol/water) ; IR (KBr) $3345(\mathrm{NH}), 1600$ $(\mathrm{CH}=\mathrm{CH}$ ), 1680, 1700, 1710 (amidic $\mathrm{C}=\mathrm{O}$ ), 1550 (C...C) of aromatic ring $\mathrm{cm}^{-1} ;{ }^{1} \mathrm{H}$ NMR $\left(\mathrm{CDCl}_{3}\right) \mathrm{d}$ 3.47 (s, 3H, Ar- $\left.\mathrm{OCH}_{3}\right), 5.75(\mathrm{~m}, 1 \mathrm{H}, \mathrm{CHCO}), 6.69$ (d, $1 \mathrm{H},-\mathrm{COCH}=), 7.50-8.45(\mathrm{~m}, 4 \mathrm{H}, \mathrm{Ar}-\mathrm{H}), 8.30$ (d, $1 \mathrm{H},=\mathrm{CH}-\mathrm{Ar}$ ), 9.28 (ss, 2H, 2NHCO) (ppm); $\mathrm{MS}: \mathrm{M}^{+}$ 288. Anal.Calcd.for $\mathrm{C}_{14} \mathrm{H}_{12} \mathrm{~N}_{2} \mathrm{O}_{5} ; \mathrm{C}, 58.33 ; \mathrm{H}, 4.16$ ; N, 9.72. Found : C, 58.32; H, 4.15 ; N, 9.76.

1-(21-Thiobarbiturinyl) -3- (ochlorophenyl) chalcone (2d). $86 \%, \mathrm{mp} 275^{\circ} \mathrm{C}$ (ethanol/water); IR (KBr) $3343(\mathrm{NH}), 1610(\mathrm{CH}=\mathrm{CH})$, 1690, 1715 (amidic (C=O), $730(\mathrm{C}-\mathrm{Cl}), 1510(\mathrm{C} \ldots \mathrm{C})$ of aromatic ring, $1031(\mathrm{C}=\mathrm{S}) \mathrm{cm}^{-1} ;{ }^{1} \mathrm{H} \mathrm{NMR}\left(\mathrm{CDCl}_{3}\right)$ d $5.83(\mathrm{~m} \mathrm{1H}, \mathrm{CHCO}), 6.73$ (d. $1 \mathrm{H},-\mathrm{COCH}=), 7.15-$ $8.00(\mathrm{~m}, 4 \mathrm{H}, \mathrm{Ar}-\mathrm{H}), 8.45$ (d, 1H, = CH-Ar), 9.29 (ss, 
Table 1: Anticonvulsant activity and toxicity data of compounds 1a-6I (Scheme 1)

\begin{tabular}{|c|c|c|c|c|c|c|c|}
\hline \multirow[t]{2}{*}{ Compd. } & \multirow[t]{2}{*}{$x$} & \multirow[t]{2}{*}{$\mathbf{R}$} & \multirow[t]{2}{*}{$\mathbf{R}^{1}$} & \multirow[t]{2}{*}{$\begin{array}{c}\text { Dose } \\
\text { (mg/kg i.p) }\end{array}$} & \multicolumn{2}{|c|}{$\begin{array}{l}\text { Anticonvulsant } \\
\text { Activity }\end{array}$} & \multirow[t]{2}{*}{$\begin{array}{c}\text { ALD50 } \\
\text { (mg/kgi.p.) }\end{array}$} \\
\hline & & & & & MES & PTZ & \\
\hline $1 a$ & $\mathrm{O}$ & - & - & 50 & 20 & 10 & $>1000$ \\
\hline $1 b$ & $\mathrm{~s}$ & - & - & 50 & 20 & 20 & $>1000$ \\
\hline $2 a$ & 0 & $2-\mathrm{Cl}$ & - & 50 & 40 & 40 & $>1000$ \\
\hline $2 b$ & 0 & $4-\mathrm{OH}$ & - & 50 & 30 & 20 & $>1000$ \\
\hline $2 c$ & 0 & $3-\mathrm{OCH}_{3}$ & - & 50 & 20 & 10 & $>1000$ \\
\hline $2 d$ & $S$ & $2-\mathrm{Cl}$ & - & 50 & 50 & 50 & $>1000$ \\
\hline $2 e$ & $S$ & $4-\mathrm{OH}$ & - & 50 & 30 & 20 & $>1000$ \\
\hline $2 f$ & $S$ & $3-\mathrm{OCH}_{3}$ & - & 50 & 40 & 30 & $>1000$ \\
\hline $3 a$ & 0 & $2-\mathrm{Cl}^{3}$ & - & 50 & 60 & 60 & $>1000$ \\
\hline $3 b$ & 0 & $4-\mathrm{OH}$ & - & 50 & 60 & 40 & $>1000$ \\
\hline $3 c$ & 0 & $3-\mathrm{OCH}_{3}$ & - & 50 & 40 & 40 & $>1000$ \\
\hline \multirow[t]{3}{*}{$3 d$} & $S$ & $2-\mathrm{Cl}^{3}$ & - & 50 & 80 & 80 & \\
\hline & & & & 2 & 40 & 30 & $>2000$ \\
\hline & & & & 17.5 & 20 & 20 & \\
\hline $3 e$ & $S$ & $4-\mathrm{OH}$ & - & 50 & 60 & 60 & $>1000$ \\
\hline $3 f$ & $S$ & $3-\mathrm{OCH}_{3}$ & - & 50 & 70 & 60 & $>1000$ \\
\hline $4 a$ & 0 & $2-\mathrm{Cl}$ & - & 50 & 50 & 50 & $>1000$ \\
\hline $4 b$ & 0 & $4-\mathrm{OH}$ & - & 50 & 40 & 30 & $>1000$ \\
\hline $4 c$ & 0 & $3-\mathrm{OCH}_{3}$ & - & 50 & 40 & 40 & $>1000$ \\
\hline $4 d$ & $S$ & $2-\mathrm{Cl}^{3}$ & - & 50 & 70 & 60 & $>1000$ \\
\hline $4 e$ & $S$ & $4-\mathrm{OH}$ & - & 50 & 60 & 50 & $>1000$ \\
\hline $4 f$ & $S$ & $3-\mathrm{OCH}_{3}$ & - & 50 & 60 & 60 & $>1000$ \\
\hline $5 a$ & 0 & $2-\mathrm{Cl}$ & $4-\mathrm{Cl}$ & 50 & 60 & 60 & $>1000$ \\
\hline $5 b$ & 0 & $2-\mathrm{Cl}$ & $4-\mathrm{OCH}_{3}$ & 50 & 70 & 70 & $>1000$ \\
\hline $5 c$ & $\mathrm{O}$ & $4-\mathrm{OH}$ & $4-\mathrm{Cl}$ & 50 & 60 & 50 & $>1000$ \\
\hline $5 d$ & 0 & $4-\mathrm{OH}$ & $4-\mathrm{OCH}_{3}$ & 50 & 60 & 50 & $>1000$ \\
\hline $5 e$ & 0 & $3-\mathrm{OCH}_{3}$ & $4-\mathrm{Cl}$ & 50 & 70 & 70 & $>1000$ \\
\hline $5 f$ & 0 & $3-\mathrm{OCH}_{3}$ & $4-\mathrm{OCH}_{3}$ & 50 & 70 & 60 & $>1000$ \\
\hline $5 g$ & $S$ & $2-\mathrm{Cl}^{3}$ & $4-\mathrm{Cl}$ & 50 & 60 & 60 & $>1000$ \\
\hline \multirow[t]{3}{*}{$5 \mathrm{~h}$} & $S$ & $2-\mathrm{Cl}$ & $4-\mathrm{OCH}_{3}$ & 50 & 90 & 90 & \\
\hline & & & & 25 & 50 & 40 & $>2000$ \\
\hline & & & & 17.5 & 20 & 20 & \\
\hline $5 i$ & $S$ & $4-\mathrm{OH}$ & $4-\mathrm{Cl}$ & 50 & 80 & 80 & $>1000$ \\
\hline $5 j$ & $S$ & $4-\mathrm{OH}$ & $4-\mathrm{OCH}_{3}$ & 50 & 80 & 70 & $>1000$ \\
\hline $5 k$ & $S$ & $3-\mathrm{OCH}_{3}$ & $4-\mathrm{Cl}$ & 50 & 70 & 60 & $>1000$ \\
\hline 51 & $\mathrm{~s}$ & $3-\mathrm{OCH}_{3}$ & $4-\mathrm{OCH}_{3}$ & 50 & 80 & 80 & $>1000$ \\
\hline $6 a$ & 0 & $2-\mathrm{Cl}^{3}$ & $4-\mathrm{Cl}^{3}$ & 50 & 50 & 40 & $>1000$ \\
\hline $6 b$ & 0 & $2-\mathrm{Cl}$ & $4-\mathrm{OCH}_{3}$ & 50 & 50 & 50 & $>1000$ \\
\hline $6 c$ & 0 & $4-\mathrm{OH}$ & $4-\mathrm{Cl}^{3}$ & 50 & 60 & 50 & $>1000$ \\
\hline $6 d$ & 0 & $4-\mathrm{OH}$ & $4-\mathrm{OCH}_{3}$ & 50 & 60 & 60 & $>1000$ \\
\hline $6 e$ & 0 & $3-\mathrm{OCH}_{3}$ & $4-\mathrm{Cl}^{3}$ & 50 & 60 & 50 & $>1000$ \\
\hline $6 f$ & 0 & $3-\mathrm{OCH}_{3}^{3}$ & $4-\mathrm{OCH}_{3}$ & 50 & 60 & 60 & $>1000$ \\
\hline $6 \mathrm{~g}$ & $\mathrm{~s}$ & $2-\mathrm{Cl}$ & $4-\mathrm{Cl}$ & 50 & 70 & 50 & $>1000$ \\
\hline
\end{tabular}




\begin{tabular}{|c|c|c|c|c|c|c|c|}
\hline $6 \mathrm{~h}$ & S & $2-\mathrm{Cl}$ & $4-\mathrm{OCH}_{3}$ & 50 & 60 & 60 & $>1000$ \\
\hline $6 i$ & S & $4-\mathrm{OH}$ & $4-\mathrm{Cl}$ & 50 & 70 & 60 & $>1000$ \\
\hline $6 j$ & S & $4-\mathrm{OH}$ & $4-\mathrm{OCH}_{3}$ & 50 & 60 & 60 & $>1000$ \\
\hline $6 \mathrm{k}$ & S & $3-\mathrm{OCH}_{3}$ & $4-\mathrm{Cl}$ & 50 & 70 & 60 & $>1000$ \\
\hline 61 & S & $3-\mathrm{OCH}_{3}$ & $4-\mathrm{OCH}_{3}$ & 50 & 60 & 50 & $>1000$ \\
\hline \multicolumn{4}{|c|}{ Phenytoin sodium } & 30 & 80 & & \\
\hline \multicolumn{4}{|c|}{ Sodium valproate } & 80 & & 80 & \\
\hline \multicolumn{4}{|c|}{ Propylene glycol } & 50 & 0 & 0 & \\
\hline
\end{tabular}

${ }^{*} \mathrm{p}<0.05, \quad{ }^{* *} \mathrm{p}<0.01, \quad{ }^{* * *} \mathrm{p}<0.001$.

2H, 2NHCO) (ppm); MS: $\mathrm{M}^{+}$308.5. Anal.Calcd.for $\mathrm{C}_{13} \mathrm{H}_{9} \mathrm{~N}_{2} \mathrm{O}_{3} \mathrm{ClS} ; \mathrm{C}, 50.56 ; \mathrm{H}, 2.91$; N, 9.07; Found : C, 50.50; H, 2.95; N, 9.01 .

1 - ( $2^{\prime}$ - Thiobarbiturinyl) - 3 - ( p hydroxyphenyl) chalcone (2e). $82 \%, \mathrm{mp} 205^{\circ} \mathrm{C}$ (DMF); IR (KBr) $3345(\mathrm{NH}), 1620(\mathrm{CH}=\mathrm{CH}), 1705$, 1715 (amidic $\mathrm{C}=\mathrm{O}), 1500(\mathrm{C} \ldots \mathrm{C})$ of aromaticring, $1060(\mathrm{C}=\mathrm{S}) \mathrm{cm}^{-1} ;{ }^{1} \mathrm{H}$ NMR $\left(\mathrm{CDCl}_{3}\right)$ d 9.15 (s, 1H. Ar$\mathrm{OH}),, 5.65(\mathrm{~m}, 1 \mathrm{H}, \mathrm{CHCO}), 6.80(\mathrm{~d}, 1 \mathrm{H},-\mathrm{COCH}=)$, 7.00-8.15 (m, 4H, Ar-H), 8.40 (d, 1H, = CH-Ar), 9.26 (ss, 2H, 2NHCO) (ppm); MS: $\mathrm{M}^{+} 290$. Anal.Calcd.for $\mathrm{C}_{13} \mathrm{H}_{10} \mathrm{~N}_{2} \mathrm{O}_{4} \mathrm{~S} ; \mathrm{C}, 53.79 ; \mathrm{H}, 3.44 ; \mathrm{N}, 9.65$; Found : $\mathrm{C}, 53.81 ; \mathrm{H}, 3.47 ; \mathrm{N}, 9.68$.

1 - ( 2 ' - Thiobarbiturinyl) - 3 - ( m methoxyphenyl) chalcone (2f). $80 \%, \mathrm{mp} 220^{\circ} \mathrm{C}$ (benzene/petroleum ether); IR (KBr) $3330(\mathrm{NH})$, $1630(\mathrm{CH}=\mathrm{CH}$ ), 1685, 1690 (amidic $\mathrm{C}=\mathrm{O}$ ), 1560 (C...C) of aromatic ring, $1030(\mathrm{C}=\mathrm{S}) \mathrm{cm}^{-1} ;{ }^{1} \mathrm{H} \mathrm{NMR}$ $\left(\mathrm{CDCl}_{3}\right)$ d $3.43\left(\mathrm{~s}, 3 \mathrm{H}, \mathrm{Ar}-\mathrm{OCH}_{3}\right), 5.60(\mathrm{~m}$, $1 \mathrm{HCHCO}), 6.75(\mathrm{~d}, 1 \mathrm{H},-\mathrm{COCH}=), 7.30-8.60(\mathrm{~m}$, $4 \mathrm{H}, \mathrm{Ar}-\mathrm{H}$ ), 8.30 (d, 1H, = CH-Ar), 9.20 (ss, 2H, 2NHCO) (ppm) ; MS : $\mathrm{M}^{+}$304. Anal.Calcd.for $\mathrm{C}_{14} \mathrm{H}_{12} \mathrm{~N}_{2} \mathrm{O}_{4} \mathrm{~S} ; \mathrm{C}, 53.79 ; \mathrm{H}, 3.44 ; \mathrm{N}, 9.65$. Found : C, 53.81; H, 3.47; N, 9.68.

4-(21-Oxobarbiturinyl)-2-(o-chlorophenyl)2, 3 - dihydro-1, 5-benzothiazepines (3a). The methanolic solution $(50 \mathrm{~mL})$ of $1-\left(2^{1}-\right.$ oxobarbiturinyl)- 3- (m-chlorophenyl)- chalcone (2a) (0.01 mol) was added 2-aminothiophenol (0.01 mol) with few drops of glacial acetic acid and refluxed for 3-5h. After refluxing solvent was distilled off under reduced pressure and the solid thus obtained was recrystallized from ethanol to give $3 a(85 \%)$, $\mathrm{mp} 148^{\circ} \mathrm{C}$; IR (KBr) $3345(\mathrm{NH}), 1690,1710,1715$ (amidic C = O), $1629(\mathrm{C}=\mathrm{C}), 1460(\mathrm{C}=\mathrm{N}), 690(\mathrm{C}-$
Cl), 671 (C-S-C) cm ${ }^{-1} ;{ }^{1} \mathrm{H}$ NMR $\left(\mathrm{CDCl}_{3}\right)$ d 9.25 (ss, $2 \mathrm{H}) ,2 \mathrm{NHCO}), 3.60(\mathrm{t}, 1 \mathrm{H},-\mathrm{CH}), 7.65\left(\mathrm{~d}, 2 \mathrm{H}, \mathrm{C}_{3}-\mathrm{H}\right.$ of thiazepine ring), 7.70-6.65 (m, 8H, Ar-H), 6.49 (t, $1 \mathrm{H}, \mathrm{C}_{2}-\mathrm{H}$ of thiazepine ring) (ppm); $\mathrm{MS}: \mathrm{M}^{+} 399.5$ .Anal. Calcd.for $\mathrm{C}_{19} \mathrm{H}_{14} \mathrm{~N}_{3} \mathrm{O}_{3} \mathrm{SCl}$; C, 57.07; H, 3.50; N, 10.51. Found : C, 57.10; H, 3.48; N, 10.55.

4 - (21-Oxobarbiturinyl) - 2- (phydroxyphenyl) -2,3 - dihydro - 1,5 benzothiazepines (3b). 78\%, mp $158^{\circ} \mathrm{C}$ (Ethanol); IR (KBr) $3320(\mathrm{NH}), 1700,1710,1715$ (amidic $\mathrm{C}=\mathrm{O}$ ), $3438(\mathrm{OH}), 1638(\mathrm{C}=\mathrm{C}), 1469(\mathrm{C}=\mathrm{N}), 680(\mathrm{C}-\mathrm{S}-\mathrm{C})$ $\mathrm{cm}^{-1} ;{ }^{1} \mathrm{H} \mathrm{NMR}\left(\mathrm{CDCl}_{3}\right) \delta 9.23$ (ss, $2 \mathrm{H}, 2 \mathrm{NHCO}$ ), $3.91(\mathrm{t}, 1 \mathrm{H},-\mathrm{CH}), 9.11(\mathrm{~s}, 1 \mathrm{H}, \mathrm{Ar}-\mathrm{OH}), 7.68$ (d, 2H, $\mathrm{C}_{3}-\mathrm{H}$ of thiazepine ring). 7.72-6.69 $(\mathrm{m}, 8 \mathrm{H}, \mathrm{Ar}-\mathrm{H})$, 6.48 (t, $1 \mathrm{H}, \mathrm{C}_{2}-\mathrm{H}$ of thiazepine ring) (ppm). MS : $\mathrm{M}^{+}$381. Anal.Calcd for $\mathrm{C}_{19} \mathrm{H}_{15} \mathrm{~N}_{3} \mathrm{O}_{4} \mathrm{~S} ; \mathrm{C}, 59.8 ; \mathrm{H}$, 3.93; N, 11.02.Found: C,59.02; H,3.95; N, 11.05.

4-(21-Oxobarbiturinyl) - $2-(\mathrm{m}-$ methoxyphenyl) - 2,3 - dihydro - 1,5 benzothiazepines (3c). 78\%, mp $146^{\circ} \mathrm{C}$ (methanol) ; IR (KBr) $3343(\mathrm{NH}), 1690,1700,1725$ (amidic $\mathrm{C}=\mathrm{O}), 1625(\mathrm{C}=\mathrm{C}), 1462(\mathrm{C}=\mathrm{N}), 1225\left(\mathrm{OCH}_{3}\right), 675$ (C-S-C) $\mathrm{cm}^{-1}$; ${ }^{1} \mathrm{H}$ NMR $\left(\mathrm{CDCl}_{3}\right)$ d 9.21 (ss, $2 \mathrm{H}$, 2NHCO), $3.62(\mathrm{t}, 1 \mathrm{H},-\mathrm{CH}), 7.75-6.71(\mathrm{~m}, 8 \mathrm{H}, \mathrm{Ar}-\mathrm{H})$, $7.65\left(\mathrm{~d}, 2 \mathrm{H}, \mathrm{C}_{3} \mathrm{H}\right.$ of thiazepine ring), $6.45\left(\mathrm{t}, 1 \mathrm{H}, \mathrm{C}_{2}-\right.$ $\mathrm{H}$ of thiazepine ring), $3.42\left(\mathrm{~s}, 3 \mathrm{H}, \mathrm{OCH}_{3}\right)(\mathrm{ppm}) ; \mathrm{MS}$ : $\mathrm{M}^{+}$411. Anal.Calcd.for $\mathrm{C}_{20} \mathrm{H}_{17} \mathrm{~N}_{3} \mathrm{O}_{5} \mathrm{~S} ; \mathrm{C}, 58.39$; $\mathrm{H}$, 4.13 ; N, 10.21. Found : C, 58.35; H, 4.15; N, 10.25.

4 - (21-Thiobarbiturinyl) - 2 - (ochlorophenyl) - 2, 3- dihydro - 1,5 -benzothiazepine (3d). $80 \%, \mathrm{mp} 155^{\circ} \mathrm{C}$ (ethanol); IR (KBr) $3330(\mathrm{NH})$, 1700, 1710 (amidic $\mathrm{C}=\mathrm{O}$ ), 1050 (C=S), 1625 (C=C), $1455(\mathrm{C}=\mathrm{N}), 680(\mathrm{C}-\mathrm{Cl}) \mathrm{cm}^{-1} ;{ }^{1} \mathrm{H} \mathrm{NMR}\left(\mathrm{CDCl}_{3}\right) 9.30$ (ss, $2 \mathrm{H}, 2 \mathrm{NHCO}), 3.57$ (t, 1H,-CH), 7.71-6.74 (m, $8 \mathrm{H}, \mathrm{Ar}-\mathrm{H}), 7.68\left(\mathrm{~d}, 2 \mathrm{H}, \mathrm{C}_{3}-\mathrm{H}\right.$ of thiazepine ring), 
$6.40\left(\mathrm{t}, 1 \mathrm{H}, \mathrm{C}_{2}-\mathrm{H}\right.$ of thiazepine ring) (ppm); $\mathrm{MS}: \mathrm{M}^{+}$ 415.5 Anal Calcd. for $\mathrm{C}_{19} \mathrm{H}_{14} \mathrm{~N}_{3} \mathrm{O}_{2} \mathrm{~S}_{2} \mathrm{Cl} ; \mathrm{C}, 54.87 ; \mathrm{H}$, 3.36 ; N, 10.10. Found : C, 54.82; H, 3.31; N, 10.13.

4 - (21-Thiobarbiturinyl) - 2 - (phydroxyphenyl) - 2,3 - dihydro - 1,5 benzothiazepine (3e). $78 \%, \mathrm{mp} 170^{\circ} \mathrm{C}$ (ethanol); IR $(\mathrm{KBr})$ 3415(NH), 1690, 1705 (amidic $\mathrm{C}=\mathrm{O}$ ), 1060 $(\mathrm{C}=\mathrm{S}), 3440(\mathrm{OH}), 1640(\mathrm{C}=\mathrm{C}), 1470(\mathrm{C}=\mathrm{N}), 688$ (C-S-C) $\mathrm{cm}^{-1} ;{ }^{1} \mathrm{H} \mathrm{NMR} \mathrm{CCDCl}_{3}$ ) $\delta 9.20$ (ss, 2H, 2NHCO), 3.62 (t, 1H,-CH), 7.70-6.65 (m, 8H, Ar-H), $9.15(\mathrm{~s}, 1 \mathrm{H}, \mathrm{Ar}-\mathrm{OH}) 7.63\left(\mathrm{~d}, 2 \mathrm{H}, \mathrm{C}_{3}-\mathrm{H}\right.$ of thiazepine ring), 6.42 (t, $1 \mathrm{H}, \mathrm{C}_{2}-\mathrm{H}$ of thiazepine ring) (ppm); $\mathrm{MS}: \mathrm{M}^{+}$397. Anal.Calcd.for $\mathrm{C}_{19} \mathrm{H}_{15} \mathrm{~N}_{3} \mathrm{O}_{3} \mathrm{~S}_{2} ; \mathrm{C}, 57.43$; $\mathrm{H}, 3.77 ; \mathrm{N}, 10.57$. Found : C,57.40; H,3.79; N, 10.60 .

4 - (2 ${ }^{1}$-Thiobarbiturinyl) - 2 - (mmethoxyphenyl) - 2,3 - dihydro - 1,5 benzothiazepine (3f). $75 \%$, mp $164^{\circ} \mathrm{C}$ (methanol); IR (KBr) $3410(\mathrm{NH}), 1700,1710$ (amidic C=O), 1055 $(\mathrm{C}=\mathrm{S}), 1629(\mathrm{C}=\mathrm{O}), 1460(\mathrm{C}=\mathrm{N}), 1230\left(\mathrm{OCH}_{3}\right), 680$ (C-S-C) $\mathrm{cm}^{-1} ;{ }^{1} \mathrm{H}$ NMR $\mathrm{CDCl}_{3}$ ) d 9.23 (ss, $2 \mathrm{H}$, 2NHCO), 3.59 (t, 1H,-CH), 7.71-6.68 (m, 8H, Ar-H), $7.60\left(\mathrm{~d}, 2 \mathrm{H}, \mathrm{C}_{3}-\mathrm{H}\right.$ of thiazepine ring), $6.48(\mathrm{t}, 1 \mathrm{H}$, $\mathrm{C}_{-}-\mathrm{H}$ of thiazepine ring) (ppm); $\mathrm{MS}: \mathrm{M}+427$. Anal.Calcd.for $\mathrm{C}_{20} \mathrm{H}_{17} \mathrm{~N}_{3} \mathrm{O}_{4} \mathrm{~S}_{2} ; \mathrm{C}, 56.20 ; \mathrm{H}, 3.98$; $\mathrm{N}, 9.83$. Found : C,56.22; H, 3.94; N,9.80.

4 - (21-Oxobarbiturinyl) - 2 - (0chlorophenyl) - 2,3 - dihydro - 1,5 -benzoxazepines (4a). The methanolic solution $(50 \mathrm{~mL})$ of $1-\left(2^{1}\right.$ oxobarbiturinyl)-3-(m-chlorophenyl)-chalcone (2a) (0.01 mol) was added 2-aminophenol $(0.01 \mathrm{~mol})$ with few drops of glacial acetic acid and refluxed for 35h. After refluxing solvent was distilled off under reduced pressure and the solid thus obtained was recrystallized from ethanol to give $4 \mathrm{a}$ (85\%), mp $150^{\circ} \mathrm{C}$; IR (KBr) $3340(\mathrm{NH}), 1680,1700,1710$ (amidic $\mathrm{C}=\mathrm{O}), 1633(\mathrm{C}=\mathrm{C}), 1470(\mathrm{C}=\mathrm{N}), 660$ (C$\mathrm{Cl}$ ), 1075 (C-O-C) cm ${ }^{-1}$; ${ }^{1} \mathrm{H}$ NMR $\left(\mathrm{CDCl}_{3}\right)$ d 9.26 (ss, $2 \mathrm{H}, 2 \mathrm{NHCO}), 3.91(\mathrm{t}, 1 \mathrm{H},-\mathrm{CH}), 7.72\left(\mathrm{~d}, 2 \mathrm{H}, \mathrm{C}_{3}-\mathrm{H}\right.$ of oxazepine ring), $6.52\left(\mathrm{t}, 1 \mathrm{H}, \mathrm{C}_{2}-\mathrm{H}\right.$ of oxazepine ring), 7.72-6.74 (m, 8H, Ar-H) (ppm) ; MS : $\mathrm{M}^{+383.5}$. Anal.Calcd.for $\mathrm{C}_{19} \mathrm{H}_{14} \mathrm{~N}_{3} \mathrm{O}_{4} \mathrm{Cl}$; C,59.45; $\mathrm{H}, 3.65$; $\mathrm{N}$, 10.95. Found : C,59.48; H,3.62; N,10.91.

4 - (21-Oxobarbiturinyl) - 2 - (phydroxyphenyl) - 2,3 - dihydro - 1,5 benzoxazepines (4b). 76\%, mp $160^{\circ} \mathrm{C}$ (ethanol) ; IR (KBr) $3347(\mathrm{NH}), 1690,1710,1715$ (amidic $\mathrm{C}=\mathrm{O}$ ),
$3444(\mathrm{OH}), 1624(\mathrm{C}=\mathrm{C}), 1474(\mathrm{C}=\mathrm{N}), 685(\mathrm{C}-\mathrm{O}-\mathrm{C})$ $\mathrm{cm}^{-1} ;{ }^{1} \mathrm{H}$ NMR $\left(\mathrm{CDCl}_{3}\right)$ d 9.30 (ss, 2H, 2NHCO), 3.85 $(\mathrm{t}, 1 \mathrm{H},-\mathrm{CH}), 7.70\left(\mathrm{~d}, 2 \mathrm{H}, \mathrm{C}_{3}-\mathrm{H}\right.$ of oxazepine ring), $6.50\left(\mathrm{t}, 1 \mathrm{H}, \mathrm{C}_{2}-\mathrm{H}\right.$ of oxazepine ring), 7.70-6.74 (m, $8 \mathrm{H}, \mathrm{Ar}-\mathrm{H}) 9.10$ (s, 1H, Ar-OH) (ppm) ; MS : M+365. Anal.Calcd.for $\mathrm{C}_{19} \mathrm{H}_{15} \mathrm{~N}_{3} \mathrm{O}_{5} ; \mathrm{C}, 62.46 ; \mathrm{H}, 4.10 ; \mathrm{N}, 11.50$. Found: $\mathrm{C}, 62.41 ; \mathrm{H}, 4.13 ; \mathrm{N}, 11.48$.

4 - (21-Oxobarbiturinyl) - 2 - (m-methoxy phenyl) - 2,3 - dihydro - 1,5 -benzoxazepines (4c). $80 \%, \mathrm{mp} 156^{\circ} \mathrm{C}$ (benzene) ; IR $(\mathrm{KBr}) 3330(\mathrm{NH})$, 1700, 1710, 1715 (amidic $\mathrm{C}=\mathrm{O}$ ), 1628 (C=C), 1465 $(\mathrm{C}=\mathrm{N}), 1230\left(\mathrm{OCH}_{3}\right), 1070(\mathrm{C}-\mathrm{O}-\mathrm{C}) \mathrm{cm}^{-1} ;{ }^{1} \mathrm{H}$ NMR $\left(\mathrm{CDCl}_{3}\right)$ d 9.28 (ss, $\left.2 \mathrm{H}, 2 \mathrm{NHCO}\right), 3.57(\mathrm{t}, 1 \mathrm{H},-\mathrm{CH})$, 7.64.6.68 (m, 8H, Ar-H) $7.68\left(\mathrm{~d}, 2 \mathrm{H}, \mathrm{C}_{3}-\mathrm{H}\right.$ of oxazepinering), 6.48 ( $\mathrm{t}, 1 \mathrm{H}, \mathrm{C}_{2}-\mathrm{H}$ of oxazepine ring), 3.48 (s, 3H, $\mathrm{OCH}_{3}$ ) (ppm); $\mathrm{MS}: \mathrm{M}^{+} 395$. Anal.Calcd.for $\mathrm{C}_{20} \mathrm{H}_{17} \mathrm{~N}_{3} \mathrm{O}$; $\mathrm{C}, 60.75 ; \mathrm{H}, 4.30 ; \mathrm{N}, 10.63$. Found : C,60.72; $\mathrm{H}, 4.33 ; \mathrm{N}, 10.67$.

4 - (21-Thiobarbiturinyl) - 2 - (0chlorophenyl) - 2,3 - dihydro - 1,5 benzoxazepine (4d). $85 \%, \mathrm{mp} 166^{\circ} \mathrm{C}$ (ethanol); IR $(\mathrm{KBr}) 3320(\mathrm{NH}), 1690,1720$ (amidic $\mathrm{C}=\mathrm{O}$ ), 1070(C=S), 1633(C=C), $1470(\mathrm{C}=\mathrm{N}), 660(\mathrm{C}-\mathrm{Cl})$ 1075 (C-O-C) cm ${ }^{-1} ;{ }^{1} \mathrm{H}$ NMR $\left(\mathrm{CDCl}_{3}\right)$ d 9.26 (ss, $2 \mathrm{H}$, 2NHCO), 3.59 (t, 1H,-CH), 7.72-6.62 (m, 8H, Ar-H), $7.70\left(\mathrm{~d}, 2 \mathrm{H}, \mathrm{C}_{3}-\mathrm{H}\right.$ of oxazepine ring), $6.52(\mathrm{t}, 1 \mathrm{H}$, $\mathrm{C}_{2}-\mathrm{H}$ of oxazepine ring) (ppm); $\mathrm{MS}: \mathrm{M}^{+} 332$. Anal.Calcd.for $\mathrm{C}_{19} \mathrm{H}_{14} \mathrm{~N}_{3} \mathrm{O}_{3} \mathrm{SCl}$; C, $68.67 \mathrm{H}, 4.21$; N, 12.65. Found : C,68.64; $\mathrm{H}, 4.24 ; \mathrm{N}, 12.67$.

4 - (21-Thiobarbiturinyl) $-2-(p-$ hydroxyphenyl) - 2,3 - dihydro - 1,5 -benzoxazepine (4e). $80 \%, \mathrm{mp} 173^{\circ} \mathrm{C}$ (ethanol); IR (KBr) $3345(\mathrm{NH})$, 1700, 1715 (amidic $\mathrm{C}=\mathrm{O}), 1080(\mathrm{C}=\mathrm{S}), 3438(\mathrm{OH})$, $1628(\mathrm{C}=\mathrm{C}), 1465(\mathrm{C}=\mathrm{N}), 1072(\mathrm{C}-\mathrm{O}-\mathrm{C}) \mathrm{cm}^{-1} ;{ }^{1} \mathrm{H}$ $\operatorname{NMR}\left(\mathrm{CDCl}_{3}\right)$ d 9.23 (ss, $\left.2 \mathrm{H}, 2 \mathrm{NHCO}\right), 3.62$ (t, $1 \mathrm{H},-$ $\mathrm{CH}), 7.72\left(\mathrm{~d}, 2 \mathrm{H}, \mathrm{C}_{3}-\mathrm{H}\right.$ of oxazepine ring), 6.50 (t, $1 \mathrm{H}, \mathrm{C}_{2}-\mathrm{H}$ of oxazepine ring), $9.15(\mathrm{~s}, 1 \mathrm{H}, \mathrm{Ar}-\mathrm{OH})$, 7.70-6.74 (m, 8H, Ar-H), (ppm); MS : $\mathrm{M}^{+} 381$. Anal Calcd.for $\mathrm{C}_{19} \mathrm{H}_{15} \mathrm{~N}_{3} \mathrm{O}_{4} \mathrm{~S} ; \mathrm{C}, 73.75 ; \mathrm{H}, 3.93 ; \mathrm{N}, 11.02$ Found: $\mathrm{C}, 73.70 ; \mathrm{H}, 3.95 ; \mathrm{N}, 11.08$.

4 - (21-Thiobarbiturinyl) - 2 - (mmethoxyphenyl) - 2,3 - dihydro - 1,5 benzoxazepine (4f). $75 \%, \mathrm{mp} 178^{\circ} \mathrm{C}$ (benzene); IR $(\mathrm{KBr}) 3347(\mathrm{NH}), 1690,1720$ (amidic $\mathrm{C}=\mathrm{O}), 1060$ $(\mathrm{C}=\mathrm{S}), 1630(\mathrm{C}=\mathrm{C}), 1470(\mathrm{C}=\mathrm{N}), 1233\left(\mathrm{OCH}_{3}\right), 1080$ (C-O-C) $\mathrm{cm}^{-1} ;{ }^{1} \mathrm{H}$ NMR $\left(\mathrm{CDCl}_{3}\right)$ d 9.33 (ss, $2 \mathrm{H}$, 
$2 \mathrm{NHCO}), 3.65(\mathrm{t}, 1 \mathrm{H},-\mathrm{CH}), 6.48\left(\mathrm{t}, 1 \mathrm{H}, \mathrm{C}_{2}-\mathrm{H}\right.$ of oxazepine ring), $7.68\left(\mathrm{~d}, 2 \mathrm{H}, \mathrm{C}_{3}-\mathrm{H}\right.$ of oxazepine ring), $3.48\left(\mathrm{ss}, 3 \mathrm{H}, \mathrm{OCH}_{3}\right), 7.65-6.71(\mathrm{~m}, 8 \mathrm{H}, \mathrm{Ar}-\mathrm{H})$, (ppm); MS : $\mathrm{M}^{+}$395. Anal.Calcd.for $\mathrm{C}_{20} \mathrm{H}_{17} \mathrm{~N}_{3} \mathrm{O}_{4} \mathrm{~S}$; $\mathrm{C}, 60.75 ; \mathrm{H}, 4.30 ; \mathrm{N}, 10.63$. Found : $\mathrm{C}, 60.78 ; \mathrm{H}, 4.26$ ; N,10.65.

4 - (2 ${ }^{1}$-Oxobarbiturinyl) -2 - (ochlorophenyl) - 3 [(p-chlorophenylamino methylene)] -2,3 - dihydro - 1,5 - benzothiazepines (5a). 62\%, mp $185^{\circ} \mathrm{C}$; IR (KBr) $3320(\mathrm{NH}), 1690$, 1710, 1715 (amidic $C=O), 1625(C=C), 1465(C=N)$, 715 (C-Cl), 678 (C-S-C) cm-1 ; ${ }^{1} \mathrm{H}$ NMR $\left(\mathrm{CDCl}_{3}\right) \mathrm{d}$ 9.25 (ss, 2H, 2NHCO), 3.60 (t, 1H,-CH), $7.62-6.74$ $(\mathrm{m}, 12 \mathrm{H}, \mathrm{Ar}-\mathrm{H}) 3.70\left(\mathrm{~d}, 1 \mathrm{H}, \quad \mathrm{C}_{3}-\mathrm{H}\right.$ of thiazepine ring), $6.45\left(\mathrm{t}, 1 \mathrm{H}, \mathrm{C}_{2}-\mathrm{H}\right.$ of thiazepine ring), 3.04 (hump, $1 \mathrm{H}, \mathrm{CH}_{2} \mathrm{NH}$ exchangeable with $\mathrm{D}_{2} \mathrm{O}$ ), 1.62 (t, $2 \mathrm{H}, \mathrm{NHCH}_{2} \mathrm{CH}=$ ) (ppm) ; $\mathrm{MS}: \mathrm{M}^{+} 467$. Anal.Calcd.for $\mathrm{C}_{26} \mathrm{H}_{19} \mathrm{~N}_{4} \mathrm{O}_{3} \mathrm{SCl}_{2} ; \mathrm{C}, 66.80 ; \mathrm{H}, 4.06$; $\mathrm{N}, 11.99$. Found: $\mathrm{C}, 66.84 ; \mathrm{H}, 4.10 ; \mathrm{N}, 11.94$.<smiles>[Y]C1NC(=O)CC(=O)N1</smiles><smiles>[R]c1ccc(C=O)cc1</smiles><smiles>[R]c1ccc(/C=C/C(=O)C2C(=O)N([Y])C(=O)N([Tl])C2=O)cc1</smiles><smiles>[R]c1ccc(C2CC(C3C(=O)NC([Y])NC3=O)=Nc3ccccc3S2)cc1</smiles><smiles>[R]c1ccc(C2CC(C3C(=O)N([Y])C([Y])N([2H])C3=O)=Nc3ccccc3O2)cc1</smiles><smiles>[R]c1ccc(NCC2Sc3ccccc3N=C2CN=C2C(=O)NC([Y])NC2=O)cc1</smiles><smiles>[R]c1ccc(NCC2Oc3ccccc3N=C2CN=C2C(=O)NC([Y])NC2=O)cc1</smiles>

Scheme 1: 
4 - (21-Oxobarbiturinyl) - 2 - (ochlorophenyl) - 3 -[(p-methoxyphenylamino methylene)] - 2,3-dihydro-1,5-benzothiazepines (5b). $70 \%, \mathrm{mp} 172^{\circ} \mathrm{C}$ (ethanol) ; IR (KBr) $3342(\mathrm{NH})$, 1710, 1715, 1720 (amidic $\mathrm{C}=\mathrm{O}$ ), $1630(\mathrm{C}=\mathrm{C}), 1462$ $(\mathrm{C}=\mathrm{N}), 720(\mathrm{C}-\mathrm{Cl}), 671,(\mathrm{C}-\mathrm{S}-\mathrm{C}), 1229\left(\mathrm{OCH}_{3}\right) \mathrm{cm}^{-1}$ ; ${ }^{1} \mathrm{H} \mathrm{NMR}\left(\mathrm{CDCl}_{3}\right)$ d 9.26 (ss, 2H, 2NHCO), 3.62 (t, $1 \mathrm{H},-\mathrm{CH}), 7.66-6.64(\mathrm{~m}, 12 \mathrm{H}, \mathrm{Ar}-\mathrm{H}) 3.72\left(\mathrm{~d}, 1 \mathrm{H}, \mathrm{C}_{3^{-}}\right.$ $\mathrm{H}$ of thiazepine ring), 7.66-6.64 (m,12H, Ar-H), 3.72 (d, $1 \mathrm{H}, \mathrm{C}_{3}-\mathrm{H}$ of thiazepine ring), $6.43\left(\mathrm{t}, 1 \mathrm{H}, \mathrm{C}_{2}-\mathrm{H}\right.$ of thiazepine ring), 3.06 (hump, $1 \mathrm{H}, \mathrm{CH}_{2} \mathrm{NH}$ exchangeable with $\left.\mathrm{D}_{2} \mathrm{O}\right), 1.65\left(\mathrm{t}, 2 \mathrm{H}, \mathrm{NHCH}_{2} \mathrm{CH}=\right)$, $3.45\left(\mathrm{~s} .3 \mathrm{H}, \mathrm{OCH}_{3}\right)$ (ppm) ; $\mathrm{MS}: \mathrm{M}^{+} 534.5$. Anal.Calcd.for $\mathrm{C}_{27} \mathrm{H}_{23} \mathrm{~N}_{4} \mathrm{O}_{4} \mathrm{SCl}$; $\mathrm{C}, 60.61 ; \mathrm{H}, 4.30$; $\mathrm{N}, 10.47$. Found: $\mathrm{C}, 60.65 ; \mathrm{H}, 4.32 ; \mathrm{N}, 10.42$.

4 - (21-Oxobarbiturinyl) $-2-(p-$ hydroxyphenyl) - 3 - $[(p-$ chlorophenylaminomethylene)]- 2,3 - dihydro - 1,5benzothiazepines (5c). $68 \%$, mp $167^{\circ} \mathrm{C}$ (methanol) ; IR (KBr) $3345(\mathrm{NH}), 1700,1715,1725$ (amidic $\mathrm{C}=\mathrm{O}$ ), $1638(\mathrm{C}=\mathrm{C}), 1465(\mathrm{C}=\mathrm{N}), 710$ (C-Cl), 678 (C-S-C), $3457(\mathrm{OH}) \mathrm{cm}^{-1} ;{ }^{1} \mathrm{H} \mathrm{NMR}\left(\mathrm{CDCl}_{3}\right)$ d 9.23 (ss, $2 \mathrm{H}$, $2 \mathrm{NHCO}), 3.68(\mathrm{t}, 1 \mathrm{H},-\mathrm{CH}), 7.64-6.60(\mathrm{~m}, 12 \mathrm{H}, \mathrm{Ar}-$ $\mathrm{H}), 3.70\left(\mathrm{~d}, 1 \mathrm{H}, \mathrm{C}_{3}-\mathrm{H}\right.$ of thiazepine ring), $6.58(\mathrm{t}, 1 \mathrm{H}$, $\mathrm{C}_{2}-\mathrm{H}$ of thiazepine ring), 3.12 (hump, $1 \mathrm{H}, \mathrm{CH}_{2} \mathrm{NH}$ exchangeable with $\left.\mathrm{D}_{2} \mathrm{O}\right), 1.62\left(\mathrm{t}, 2 \mathrm{H}, \mathrm{NHCH}_{2} \mathrm{CH}=\right)$ 9.15 (s, $1 \mathrm{H}, \mathrm{Ar}-\mathrm{OH})$ (ppm) ; MS : $\mathrm{M}^{+} 456.5$ Anal. Calcd.for $\mathrm{C}_{26} \mathrm{H}_{21} \mathrm{~N}_{4} \mathrm{O}_{4} \mathrm{SCl} ; \mathrm{C}, 68.34 ; \mathrm{H}, 4.60 ; \mathrm{N}, 12.26$. Found: C,68.30; H,4.62; N,12.22.

4 - (21-Oxobarbiturinyl) $-2-(p-$ hydroxyphenyl) $-3-$ [(p-methoxyphenylamino methylene)] - 2,3 - dihydro - 1,5 - benzothiazepines (5d). 54\%, mp $184^{\circ} \mathrm{C}$ (DMF/Water) ; IR (KBr) 3330 $(\mathrm{NH}), 3460(\mathrm{OH}), 1680,1700,1715$ (amidic $\mathrm{C}=\mathrm{O}$ ), $1645(\mathrm{C}=\mathrm{C}), 1480(\mathrm{C}=\mathrm{N}), 682(\mathrm{C}-\mathrm{S}-\mathrm{C}), \mathrm{cm}^{-1} ;{ }^{1} \mathrm{H}$ NMR $\left(\mathrm{CDCl}_{3}\right)$ d 7.64-6.62 (m, 12H, Ar-H), 9.27 (ss, $2 \mathrm{H}, 2 \mathrm{NHCO}), 3.57(\mathrm{t}, 1 \mathrm{H} . \mathrm{CH}-), 3.75,\left(\mathrm{~d}, 1 \mathrm{H}, \mathrm{C}_{3}-\mathrm{H}\right.$ of thiazepine ring), $6.41\left(\mathrm{t}, 1 \mathrm{H}, \mathrm{C}_{2}-\mathrm{H}\right.$ of thiazepine ring), $3.44\left(\mathrm{~s}, 3 \mathrm{H}, \mathrm{OCH}_{3}\right), \quad(\quad, 1 \mathrm{H}, \mathrm{Ar}-\mathrm{OH}), 3.10$ (hump, $1 \mathrm{H}, \mathrm{CH}_{2} \mathrm{NH}$ exchangeable with $\mathrm{D}_{2} \mathrm{O}$ ), 1.60 (t, $2 \mathrm{H}$, $\left.\mathrm{NHCH}_{2} \mathrm{CH}=\right)$ (ppm); $\mathrm{MS}: \mathrm{M}^{+} 516$ Anal. Calcd.for $\mathrm{C}_{27} \mathrm{H}_{24} \mathrm{~N}_{4} \mathrm{O}_{5} \mathrm{~S} ; \mathrm{C}, 62.79 ; \mathrm{H}, 4.65 ; \mathrm{N}, 10.85$. Found: $\mathrm{C}, 62.76 ; \mathrm{H}, 4.63 ; \mathrm{N}, 10.88$.

$4-\left(2^{1}\right.$-Oxobarbiturinyl $)-2-(\mathrm{m}-$ methoxyphenyl) - 3 - [(p-chlorophenylamino methylene)]- 2,3-dihydro-1,5-benzothiazepines (5e). 52\%, mp $173^{\circ} \mathrm{C}$ (benzene); IR (KBr) $3360(\mathrm{NH})$,
1670, 1700, 1720 (amidic C=O), 1650 (C=C), 1465 $(\mathrm{C}=\mathrm{N}), 710(\mathrm{C}-\mathrm{Cl}), 688(\mathrm{C}-\mathrm{S}-\mathrm{C}), \mathrm{cm}^{-1} ;{ }^{1} \mathrm{H}$ NMR $\left(\mathrm{CDCl}_{3}\right)$ d 9.30 (ss, $\left.2 \mathrm{H}, 2 \mathrm{NHCO}\right), 3.55(\mathrm{t}, 1 \mathrm{H}, \mathrm{CH}-)$, 7.65-6.78 $(\mathrm{m}, 12 \mathrm{H}, \mathrm{Ar}-\mathrm{H}) 3.72\left(\mathrm{~d}, 1 \mathrm{H}, \mathrm{C}_{3}-\mathrm{H}\right.$ of thiazepine ring), $6.55\left(\mathrm{t}, 1 \mathrm{H}, \mathrm{C}_{2}-\mathrm{H}\right.$ of thiazepine ring), $3.35\left(\mathrm{~s}, 3 \mathrm{H}, \mathrm{OCH}_{3}\right), 3.10$ (hump, $1 \mathrm{H}, \mathrm{CH}_{2} \mathrm{NH}$ exchangeable with $\left.\mathrm{D}_{2} \mathrm{O}\right), 1.68\left(\mathrm{t}, 2 \mathrm{H}, \mathrm{NHCH}_{2} \mathrm{CH}=\right)$ (ppm) ; $\mathrm{MS}: \mathrm{M}^{+} 534.5$ Anal. Calcd.for $\mathrm{C}_{27} \mathrm{H}_{23} \mathrm{~N}_{4} \mathrm{O}_{4} \mathrm{SCl}$; $\mathrm{C}, 60.61 ; \mathrm{H}, 4.30 ; \mathrm{N}, 10.47$. Found : $\mathrm{C}, 60.63 ; \mathrm{H}, 4.28$; $\mathrm{N}, 10.43$.

4 - (21-Oxobarbiturinyl $)-2-(m-$ methoxyphenyl) - 3 - [(p-methoxyphenylamino methylene)]- 2,3 - dihydro - 1,5 - benzothiazepines (5f). $67 \%, \mathrm{mp} 168^{\circ} \mathrm{C}$ (DMF/Water); IR (KBr) 3347 $(\mathrm{NH}), 1700,1710,1720$ (amidic $\mathrm{C}=\mathrm{O}), 3432(\mathrm{OH})$, $1618(\mathrm{C}=\mathrm{C}), 1458(\mathrm{C}=\mathrm{N}), 1221\left(\mathrm{OCH}_{3}\right), 665$ (C-SC), $\mathrm{cm}^{-1} ;{ }^{1} \mathrm{H}$ NMR $\left(\mathrm{CDCl}_{3}\right)$ d 9.29 (ss, $2 \mathrm{H}, 2 \mathrm{NHCO}$ ), $3.59(\mathrm{t}, 1 \mathrm{H}, \mathrm{CH}-), 7.68-6.80(\mathrm{~m}, 12 \mathrm{H}, \mathrm{Ar}-\mathrm{H}) 3.70(\mathrm{~d}$, $1 \mathrm{H}, \mathrm{C}_{3}-\mathrm{H}$ of thiazepine ring), $6.40\left(\mathrm{t}, 1 \mathrm{H}, \mathrm{C}_{2}-\mathrm{H}\right.$ of thiazepine ring), 6.40 (t, $1 \mathrm{H}, \mathrm{C}_{2}-\mathrm{H}$ of thiazepine ring), $3.41\left(\mathrm{~s}, 6 \mathrm{H}, 2 \times \mathrm{OCH}_{3}\right.$ ). 3.00 (hump, $1 \mathrm{H}, \mathrm{CH}_{2} \mathrm{NH}$ exchangeable with $\left.\mathrm{D}_{2} \mathrm{O}\right), 1.59\left(\mathrm{t}, 2 \mathrm{H}, \mathrm{NHCH}_{2} \mathrm{CH}=\right)$ (ppm) ; MS : $\mathrm{M}^{+} 530$ Anal. Calcd.for $\mathrm{C}_{28} \mathrm{H}_{26} \mathrm{~N}_{4} \mathrm{O}_{5} \mathrm{~S}$; $\mathrm{C}, 63.39 ; \mathrm{H}, 4.90 ; \mathrm{N}, 10.56$. Found : $\mathrm{C}, 63.41 ; \mathrm{H}, 4.88$ ; N, 10.58 .

4 - (21-Thiobarbiturinyl) - 2 - (ochlorophenyl) - 3 - [(p-chlorophenylamino methylene)]- 2,3 - dihydro - 1,5 - benzothiazepines (5g). $53 \%, \mathrm{mp} 177^{\circ} \mathrm{C}$ (ethanol); IR ( $\left.\mathrm{KBr}\right) 3328(\mathrm{NH})$, 1680, 1710, (amidic $C=O), 1620(C=C), 1440(C=C)$, $720(\mathrm{C}=\mathrm{Cl}), 690(\mathrm{C}-\mathrm{S}-\mathrm{C}), 1130(\mathrm{C}=\mathrm{S}) \mathrm{cm}^{-1} ;{ }^{1} \mathrm{H}$ NMR $\left(\mathrm{CDCl}_{3}\right) \mathrm{d} 9.27(\mathrm{ss}, 2 \mathrm{H}, 2 \mathrm{NHCO}), 3.65(\mathrm{t}, 1 \mathrm{H}, \mathrm{CH}-)$, 7.65- $6.74(\mathrm{~m}, 12 \mathrm{H}, \mathrm{Ar}-\mathrm{H}) 3.72\left(\mathrm{~d}, 1 \mathrm{H}, \mathrm{C}_{3}-\mathrm{H}\right.$ of thiazepine ring), 6.55 (t, $1 \mathrm{H}, \mathrm{C}_{2}-\mathrm{H}$ of thiazepine ring), 3.10 (hump, $1 \mathrm{H}, \mathrm{CH}_{2} \mathrm{NH}$ exchangeable with $\mathrm{D}_{2} \mathrm{O}$ ), $1.65\left(\mathrm{t}, 2 \mathrm{H}, \mathrm{NHCH}_{2} \mathrm{CH}=\right)$ (ppm) ; $\mathrm{MS}: \mathrm{M}^{+} 554$ Anal. Calcd.for $\mathrm{C}_{26} \mathrm{H}_{19} \mathrm{~N}_{4} \mathrm{O}_{2} \mathrm{~S}_{2} \mathrm{Cl}_{2} ; \mathrm{C}, 56.31 ; \mathrm{H}, 3.42$; $\mathrm{N}, 10.10$. Found: $\mathrm{C}, 56.33$; H,3.45; N,10.14.

4 - (21-Thiobarbiturinyl) - 2 - (ochlorophenyl) - 3 - [(p-chlorophenylamino methylene)]- 2,3 - dihydro - 1,5 - benzothiazepines (5h). $44 \%, \mathrm{mp} 183^{\circ} \mathrm{C}$ (ethanol); IR (KBr) $3347(\mathrm{NH})$, 1700, 1710, (amidic $C=O), 1628(C=C), 1490(C=N)$, $730(\mathrm{C}=\mathrm{Cl}), 675(\mathrm{C}-\mathrm{S}-\mathrm{C}), 1235\left(\mathrm{OCH}_{3}\right) 1127(\mathrm{C}=\mathrm{S})$ $\mathrm{cm}^{-1} ;{ }^{1} \mathrm{H} \mathrm{NMR}\left(\mathrm{CDCl}_{3}\right)$ d 9.30 (ss, $2 \mathrm{H}, 2 \mathrm{NHCO}$ ), 3.68 $(\mathrm{t}, 1 \mathrm{H}, \mathrm{CH}-), 7.68-6.64(\mathrm{~m}, 12 \mathrm{H}, \mathrm{Ar}-\mathrm{H}) 3.70(\mathrm{~d}, 1 \mathrm{H}$, $\mathrm{C}_{3}-\mathrm{H}$ of thiazepine ring), $6.58\left(\mathrm{t}, 1 \mathrm{H}, \mathrm{C}_{2}-\mathrm{H}\right.$ of 
thiazepine ring), 3.08 (hump, $1 \mathrm{H}, \mathrm{CH}_{2} \mathrm{NH}$ exchangeable with $\mathrm{D}_{2} \mathrm{O}$ ), 1.70 (t, $2 \mathrm{H}, \mathrm{NHCH}_{2} \mathrm{CH}=$ ), $\left.3.52\left(\mathrm{~s}, 3 \mathrm{H}, \mathrm{OCH}_{3}\right), \mathrm{ppm}\right)$; $\mathrm{MS}: \mathrm{M}^{+} 550.5$ Anal. Calcd.for $\mathrm{C}_{27} \mathrm{H}_{23} \mathrm{~N}_{4} \mathrm{O}_{3} \mathrm{~S}_{2} \mathrm{Cl} ; \mathrm{C}, 58.85 ; \mathrm{H}, 4.17$; $\mathrm{N}, 10.17$. Found : C, $58.82 ; \mathrm{H}, 4.19 ; \mathrm{N}, 10.20$.

4 - (21-Thiobarbiturinyl) $-2-(p-$ hydroxyphenyl) - 3- [(p-chlorophenylamino methylene)]-2,3-dihydro-1,5-benzothiazepines (5i). $55 \%, \mathrm{mp} 163^{\circ} \mathrm{C}$ (methanol); IR (KBr) $3348(\mathrm{NH})$, 1690, 1700, (amidic $C=O), 1133(C=S), 1648(C=C)$, $1478(\mathrm{C}=\mathrm{N}), 760(\mathrm{C}-\mathrm{Cl}), 680$ (C-S-C), $3460(\mathrm{OH})$ $\mathrm{cm}^{-1} ;{ }^{1} \mathrm{H}$ NMR $\left(\mathrm{CDCl}_{3}\right)$ d 9.26 (ss, $2 \mathrm{H}, 2 \mathrm{NHCO}$ ), $3.75(\mathrm{t}, 1 \mathrm{H}, \mathrm{CH}), 7.65-6.74(\mathrm{~m}, 12 \mathrm{H}, \mathrm{Ar}-\mathrm{H}), 3.70(\mathrm{~d}$, $1 \mathrm{H}, \mathrm{C}_{3}-\mathrm{H}$ of thiazepine ring), $6.65\left(\mathrm{t}, 1 \mathrm{H}, \mathrm{C}_{2}-\mathrm{H}\right.$ of thiazepine ring), 3.15 (hump, $1 \mathrm{H}, \mathrm{CH}_{2} \mathrm{NH}$ exchangeable with $\left.\mathrm{D}_{2} \mathrm{O}\right), 1.68\left(\mathrm{t}, 2 \mathrm{H}, \mathrm{NHCH}_{2} \mathrm{CH}=\right)$, 9.10 (s, 1H, Ar-OH) (ppm); MS : $\mathrm{M}^{+} 536.5$ Anal. Calcd.for $\mathrm{C}_{26} \mathrm{H}_{21} \mathrm{~N}_{4} \mathrm{O}_{3} \mathrm{~S} 2 \mathrm{Cl}$; C, 58.15 ; $\mathrm{H}, 3.91$; $\mathrm{N}, 10.43$. Found : C,58.11; H,3.94; N, 10.45.

$4-\left(2^{1}\right.$-Thiobarbiturinyl $)-2-(p-$ hydroxyphenyl) - $3-$ [(p-methoxyphenylamino methylene)]-2,3-dihydro-1,5-benzothiazepines (5j). $68 \%, \mathrm{mp} 185^{\circ} \mathrm{C}$ (DMF/Water); IR (KBr) $3328(\mathrm{NH})$, $3465(\mathrm{OH}), 1680,1700$ (amidic $\mathrm{C}=\mathrm{O}), 1650(\mathrm{C}=\mathrm{C})$, $1470(\mathrm{C}=\mathrm{N}), 1128(\mathrm{C}=\mathrm{S}), 680(\mathrm{C}-\mathrm{S}-\mathrm{C}), \mathrm{cm}^{-1} ;{ }^{1} \mathrm{H}$ NMR $\left(\mathrm{CDCl}_{3}\right)$ d 7.60-6.75 (m, 2H, Ar-H), 9.30 (ss, $2 \mathrm{H}, 2 \mathrm{NHCO}), 3.52(\mathrm{t}, 1 \mathrm{H}, \mathrm{CH}-), 3.75\left(\mathrm{~d}, 1 \mathrm{H}, \mathrm{C}_{3}-\mathrm{H}\right.$ of thiazepine ring), $6.45\left(\mathrm{t}, 1 \mathrm{H}, \mathrm{C}_{2}-\mathrm{H}\right.$ of thiazepine ring), $3.48\left(\mathrm{~S}, 3 \mathrm{H}, \mathrm{OCH}_{3}\right) 9.11(\mathrm{~s}, 1 \mathrm{H}, \mathrm{Ar}-\mathrm{OH}), 3.13$ (hump, $1 \mathrm{H}, \mathrm{CH}_{2} \mathrm{NH}$ exchangeable with $\mathrm{D}_{2} \mathrm{O}$ ), 1.65 (t, $2 \mathrm{H}, \mathrm{NHCH}_{2} \mathrm{CH}=$ ), (ppm) ; $\mathrm{MS}: \mathrm{M}^{+} 532$ Anal. Calcd.for $\mathrm{C}_{27} \mathrm{H}_{24} \mathrm{~N}_{4} \mathrm{O}_{4} \mathrm{~S}_{2} ; \mathrm{C}, 60.90 ; \mathrm{H}, 4.51 ; \mathrm{N}, 10.52$ . Found: $\mathrm{C}, 60.92 ; \mathrm{H}, 4.48 ; \mathrm{N}, 10.54$.

4 - (21-Thiobarbiturinyl) - 2 - (mmethoxyphenyl) - 3 - [(p-chlorophenylamino methylene)]- 2,3 - dihydro -1,5-benzothiazepines (5k). $60 \%, \mathrm{mp} 162^{\circ} \mathrm{C}$ (benzene); IR (KBr) $3350(\mathrm{NH})$, 1700, 1720 (amidic $C=O), 1145(C=S), 1653(C=C)$, $1470(\mathrm{C}=\mathrm{N}), 730$ (C-Cl), 690 (C-S-C), $\mathrm{cm}^{-1} ;{ }^{1} \mathrm{H}$ $\operatorname{NMR}\left(\mathrm{CDCl}_{3}\right)$ d 9.36 (ss, 2H, 2NHCO), $3.48(\mathrm{t}, 1 \mathrm{H}$, $\mathrm{CH}-), 7.68-6.60(\mathrm{~m}, 12 \mathrm{H}, \mathrm{Ar}-\mathrm{H}), 3.70\left(\mathrm{~d}, 1 \mathrm{H}, \mathrm{C}_{3}-\mathrm{H}\right.$ of thiazepine ring), $3.38\left(\mathrm{~s}, 3 \mathrm{H}, \mathrm{OCH}_{3}\right) 3.08$ (hump, $1 \mathrm{H}, \mathrm{CH}_{2} \mathrm{NH}$ exchangeable with $\left.\mathrm{D}_{2} \mathrm{O}\right), 1.64(\mathrm{t}, 2 \mathrm{H}$, $\left.\mathrm{NHCH}_{2} \mathrm{CH}=\right)(\mathrm{ppm}) ; \mathrm{MS}: \mathrm{M}^{+}$550.5. Anal.Calcd.for $\mathrm{C}_{27} \mathrm{H}_{23} \mathrm{~N}_{4} \mathrm{O}_{3} \mathrm{~S}_{2} \mathrm{Cl} ; \mathrm{C}, 58.85 ; \mathrm{H}, 4.17 ; \mathrm{N}, 10.17$. Found : $\mathrm{C}, 58.88 ; \mathrm{H}, 4.12 ; \mathrm{N}, 10.14$.
4 - (21-Thiobarbiturinyl) - 2 - (mmethoxyphenyl) - 3 - [(p-methoxyphenylamino methylene)]- 2,3-dihydro-1,5-benzothiazepines (5I). $70 \%$, mp $182^{\circ} \mathrm{C}$ (DMF/Water); IR (KBr) $3350(\mathrm{NH})$, 1710, 1715 (amidic $\mathrm{C}=\mathrm{O}), 1150(\mathrm{C}=\mathrm{S}), 3435(\mathrm{OH})$, $1620(\mathrm{C}=\mathrm{C}), 1470(\mathrm{C}=\mathrm{N}), 1220\left(\mathrm{OCH}_{3}\right), 670$ (C-S$\mathrm{C}), \mathrm{cm}^{-1} ;{ }^{1} \mathrm{H}$ NMR $\left(\mathrm{CDCl}_{3}\right)$ d 9.23 (ss, $2 \mathrm{H}, 2 \mathrm{NHCO}$ ), $3.63(\mathrm{t}, 1 \mathrm{H}, \mathrm{CH}-), 7.62-6.60(\mathrm{~m}, 12 \mathrm{H}, \mathrm{Ar}-\mathrm{H}), 3.71(\mathrm{~d}$, $1 \mathrm{H}, \mathrm{C}_{3}-\mathrm{H}$ of thiazepine ring), $6.43\left(\mathrm{t}, 1 \mathrm{H}, \mathrm{C}_{2}-\mathrm{H}\right.$ of thiazepine ring), $3.44\left(\mathrm{~s}, 6 \mathrm{H}, 2 \mathrm{xOCH}_{3}\right), 3.04$ (hump, $1 \mathrm{H}, \mathrm{CH}_{2} \mathrm{NH}$ exchangeable with $\left.\mathrm{D}_{2} \mathrm{O}\right), 1.62(\mathrm{t}, 2 \mathrm{H}$, $\left.\mathrm{NHCH}_{2} \mathrm{CH}=\right)(\mathrm{ppm})$; $\mathrm{MS}: \mathrm{M}^{+}$546. Anal.Calcd.for $\mathrm{C}_{28} \mathrm{H}_{26} \mathrm{~N}_{4} \mathrm{O}_{4} \mathrm{~S}_{2} ; \mathrm{C}, 61.53 ; \mathrm{H}, 4.76 ; \mathrm{N}, 10.25$. Found: $\mathrm{C}, 61.55 ; \mathrm{H}, 4.79 ; \mathrm{N}, 10.21$.

4 - (21-Oxobarbiturinyl) - 2 - (ochlorophenyl) - 3 - [(p-Chlorophenylamino methylene)]- 2,3 - dihydro - 1,5 - benzoxazepines (6a). $65 \%, \mathrm{mp} 189^{\circ} \mathrm{C}$ (ethanol); IR ( $\left.\mathrm{KBr}\right) 3343(\mathrm{NH})$, 1690, 1710, 1720 (amidic $C=O), 1630(C=C), 1469$ $(\mathrm{C}=\mathrm{N}), 1040$ (C-O-C), 715 (C=Cl), $\mathrm{cm}^{-1}$; ${ }^{1} \mathrm{H}$ NMR $\left(\mathrm{CDCl}_{3}\right)$ d 9.29 (ss, 2H, 2NHCO), 3.58 (t, $1 \mathrm{H},-\mathrm{CH}$ ), 7.60-6.68(m, $12 \mathrm{H}, A r-\mathrm{H}), 3.72\left(\mathrm{~d}, 1 \mathrm{H}, \mathrm{C}_{3}-\mathrm{H}\right.$ of thiazepine ring), $6.50\left(\mathrm{t}, 1 \mathrm{H}, \mathrm{C}_{2}-\mathrm{H}\right.$ of thiazepine ring), 3.08 (hump, $1 \mathrm{H}, \mathrm{CH}_{2} \mathrm{NH}$ exchangeable with $\mathrm{D}_{2} \mathrm{O}$ ), 1.65 (t, 2H, $\left.\mathrm{NHCH}_{2} \mathrm{CH}=\right)$ (ppm) ; $\mathrm{MS}: \mathrm{M}^{+} 522$. Anal.Calcd.for $\mathrm{C}_{26} \mathrm{H}_{19} \mathrm{~N}_{4} \mathrm{O}_{4} \mathrm{Cl}_{2} ; \mathrm{C}, 59.77 ; \mathrm{H}, 3.63$; $\mathrm{N}, 10.72$. Found: $\mathrm{C}, 59.74 ; \mathrm{H}, 3.65 ; \mathrm{N}, 10.70$.

4 - (21-Oxobarbiturinyl) - 2 - (ochlorophenyl) $-3-[(p-m e t h o x y p h e n y l a m i n o$ methylene)]- 2,3-dihydro-1,5-benzoxazepines (6b). $55 \%, \mathrm{mp} 195^{\circ} \mathrm{C}$ (benzene); IR (KBr) $3350(\mathrm{NH})$, 1700, 1710, 1715 (amidic C=O), 1637 (C-C), 1469 $(\mathrm{C}=\mathrm{N}), 715(\mathrm{C}-\mathrm{Cl}), 1035(\mathrm{C}-\mathrm{O}-\mathrm{C}), 1221\left(\mathrm{OCH}_{3}\right) \mathrm{cm}^{-}$ 1 ; ${ }^{1} \mathrm{H} \mathrm{NMR}\left(\mathrm{CDCl}_{3}\right)$ d 9.24 (ss, 2H, 2NHCO), 3.60 (t, $1 \mathrm{H},-\mathrm{CH}), 7.65-6.74(\mathrm{~m}, 12 \mathrm{H}, \mathrm{Ar}-\mathrm{H}), 3.70(\mathrm{~d}, 1 \mathrm{H}$, $\mathrm{C}_{3}-\mathrm{H}$ of oxazepine ring), $6.59\left(\mathrm{t}, 1 \mathrm{H}, \mathrm{C}_{2}-\mathrm{H}\right.$ of oxazepine ring), 3.12 (hump, $1 \mathrm{H}, \mathrm{CH}_{2} \mathrm{NH}$ exchangeable with $\left.\mathrm{D}_{2} \mathrm{O}\right), 1.62\left(\mathrm{t}, 2 \mathrm{H}, \mathrm{NHCH}_{2} \mathrm{CH}=\right)$, 3.39 (s. $3 \mathrm{H}, \mathrm{OCH}_{3}$ ) (ppm) ; $\mathrm{MS}: \mathrm{M}^{+} 518.5$ Anal. Calcd.for $\mathrm{C}_{27} \mathrm{H}_{23} \mathrm{~N}_{4} \mathrm{O}_{5} \mathrm{Cl} ; \mathrm{C}, 62.48 ; \mathrm{H}, 4.43 ; \mathrm{N}, 10.80$. Found: C,62.46; H,4.47; N,10.78.

4 - (21-Oxobarbiturinyl) $-2-(p-$ hydroxyphenyl) - 3 - [(p-chlorophenylamino methylene)]- 2,3-dihydro-1,5-benzoxazepines (6c). $61 \%, \mathrm{mp} 200^{\circ} \mathrm{C}$ (methanol); IR (KBr) $3353(\mathrm{NH})$, 1700, 1712, 1717 (amidic $\mathrm{C}=\mathrm{O}$ ), $1634(\mathrm{C}=\mathrm{C}), 1470$ 
$(\mathrm{C}=\mathrm{N}), 720(\mathrm{C}-\mathrm{Cl}), 1045$ (C-O-C), $3468(\mathrm{OH}) \mathrm{cm}^{-1}$; ${ }^{1} \mathrm{H}$ NMR $\left(\mathrm{CDCl}_{3}\right)$ d 9.30 (ss, 2H, 2NHCO), 3.57 (t, $1 \mathrm{H},-\mathrm{CH}), 7.78-6.60(\mathrm{~m}, 12 \mathrm{~J} . \mathrm{Ar}-\mathrm{H}), 3.75\left(\mathrm{~d}, 1 \mathrm{H}, \mathrm{C}_{3}-\right.$ $\mathrm{H}$ of oxazepine ring), 6.61 (t, $1 \mathrm{H}, \mathrm{C}_{2}-\mathrm{H}$ of oxazepine ring), 3.16 (hump, $1 \mathrm{H}, \mathrm{CH}_{2} \mathrm{NH}$ exchangeable with $\left.\mathrm{D}_{2} \mathrm{O}\right), 1.70$ (t, $\left.2 \mathrm{H}, \mathrm{NHCH}_{2} \mathrm{CH}=\right), 9.15(\mathrm{~s}, 1 \mathrm{H}, \mathrm{OH}-$ Ar), (ppm) ; MS : $\mathrm{M}^{+}$504.5. Anal.Calcd.for $\mathrm{C}_{26} \mathrm{H}_{21} \mathrm{~N}_{4} \mathrm{O}_{5} \mathrm{Cl} ; \mathrm{C}, 61.84 ; \mathrm{H}, 4.16 ; \mathrm{N}, 11.10$. Found : $\mathrm{C}, 61.80 ; \mathrm{H}, 4.18 ; \mathrm{N}, 11.13$.

4 - (21-Oxobarbiturinyl) $-2-(p-$ hydroxyphenyl) $-3-[(p-m e t h o x y p h e n y l a m i n o$ methylene)]- 2,3-dihydro-1,5-benzoxazepines (6d). $48 \%, \mathrm{mp} 207^{\circ} \mathrm{C}$ (methanol); IR (KBr) $3347(\mathrm{NH})$, $3458(\mathrm{OH}), 1690,1700,1710$ (amidic $\mathrm{C}=\mathrm{O}$ ), 1630 $(\mathrm{C}=\mathrm{C}), 1490(\mathrm{C}=\mathrm{N}), 1035$ (C-O-C), $\mathrm{cm}^{-1}$; ${ }^{1} \mathrm{H}$ NMR $\left(\mathrm{CDCl}_{3}\right)$ d 7.80-6.85 (m, 12H, Ar-H), 9.35 (ss, 2H, $2 \mathrm{NHCO}), 3.60(\mathrm{t}, 1 \mathrm{H}, \mathrm{CH}-), 3.75\left(\mathrm{~d}, 1 \mathrm{H}, \mathrm{C}_{3}-\mathrm{H}\right.$ of oxazepine ring), $6.61\left(\mathrm{t}, 1 \mathrm{H}, \mathrm{C}_{2}-\mathrm{H}\right.$ of oxazepine ring), $3.31\left(\mathrm{~s}, 3 \mathrm{H}, \mathrm{OCH}_{3}\right.$ ), 9.10 (s, $1 \mathrm{H}, \mathrm{Ar}-\mathrm{OH}$ ), 3.12 (hump, $1 \mathrm{H}, \mathrm{CH}_{2} \mathrm{NH}$ exchangeable with $\left.\mathrm{D}_{2} \mathrm{O}\right), 1.62(\mathrm{t}, 2 \mathrm{H}$, $\left.\mathrm{NHCH}_{2} \mathrm{CH}=\right)(\mathrm{ppm}) ; \mathrm{MS}: \mathrm{M}^{+}$500. Anal.Calcd.for $\mathrm{C}_{27} \mathrm{H}_{24} \mathrm{~N}_{4} \mathrm{O}_{6} ; \mathrm{C}, 62.4 ; \mathrm{H}, 4.8 ; \mathrm{N}, 11.2$. Found: $\mathrm{C}, 62.8$; $\mathrm{H}, 4.4 ; \mathrm{N}, 11.4$.

4 - (21-Oxobarbiturinyl $)-2-(m-$ methoxyphenyl) - 3 - [(p-chlorophenylamino methylene)]- 2,3-dihydro-1,5-benzoxazepines (6e). $58 \%, \mathrm{mp} 192^{\circ} \mathrm{C}$ (DMF/Water); IR (KBr) $3350(\mathrm{NH})$, 1670, 1700, 1720 (amidic $C=O), 1647(C=C), 1460$ $(\mathrm{C}=\mathrm{N}), 715$ (C-Cl), 1025 (C-O-C), $\mathrm{cm}^{-1}$; ${ }^{1} \mathrm{H}$ NMR $\left(\mathrm{CDCl}_{3}\right)$ d 9.36 (ss, $\left.2 \mathrm{H}, 2 \mathrm{NHCO}\right), 3.50$. (t, $\left.1 \mathrm{H}, \mathrm{CH}-\right)$ 7.85-6.70 (m, 12H, Ar-H) $3.77\left(\mathrm{~d}, 1 \mathrm{H}, \mathrm{C}_{3}-\mathrm{H}\right.$ of oxazepine ring), $6.40\left(\mathrm{t}, 1 \mathrm{H}, \mathrm{C}_{2}-\mathrm{H}\right.$ of oxazepine ring), 3.02 (hump, $1 \mathrm{H}, \mathrm{CH}_{2} \mathrm{NH}$ exchangeable with $\mathrm{D}_{2} \mathrm{O}$ ), 1.60 (t, $2 \mathrm{H}, \mathrm{NHCH}_{2} \mathrm{CH}=$ ) (ppm) ; $\mathrm{MS}: \mathrm{M}^{+} 518.5$ Anal. Calcd.for $\mathrm{C}_{27} \mathrm{H}_{23} \mathrm{~N}_{4} \mathrm{O}_{5} \mathrm{Cl} ; \mathrm{C}, 62.48 ; \mathrm{H}, 4.43 ; \mathrm{N}, 10.80$. Found: $\mathrm{C}, 62.44 ; \mathrm{H}, 4.45 ; \mathrm{N}, 10.77$.

$4-\left(2^{1}\right.$-Oxobarbiturinyl $)-2-(m-$ methoxyphenyl) - 3 - [(p-methoxyphenylamino methylene)]- 2,3-dihydro-1,5-benzoxazepines (6f). $61 \%, \mathrm{mp} 198^{\circ} \mathrm{C}$ (ethanol); IR (KBr) $3350(\mathrm{NH}), 1700$, 1710, 1720 (amidic $\mathrm{C}=\mathrm{O}), 3434(\mathrm{OH}), 1618(\mathrm{C}=\mathrm{C})$, $1458(\mathrm{C}=\mathrm{N}), 1221\left(\mathrm{OCH}_{3}\right), 1030(\mathrm{C}-\mathrm{O}-\mathrm{C}), \mathrm{cm}^{-1} ;{ }^{1} \mathrm{H}$ $\operatorname{NMR}\left(\mathrm{CDCl}_{3}\right)$ d $9.40(\mathrm{ss}, 2 \mathrm{H}, 2 \mathrm{NHCO}), 3.56(\mathrm{t}, 1 \mathrm{H}$, $\mathrm{CH}-), 7.80-6.74(\mathrm{~m}, 12 \mathrm{H}, \mathrm{Ar}-\mathrm{H}) .3 .70\left(\mathrm{~d}, 1 \mathrm{H}, \mathrm{C}_{3}-\mathrm{H}\right.$ of oxazepine ring), $6.40\left(\mathrm{t}, 1 \mathrm{H}, \mathrm{C}_{2}-\mathrm{H}\right.$ of oxazepine ring), 3.41 (s, $6 \mathrm{H}, 2 \mathrm{xOCH}_{3}$ ), 3.00 (hump, $1 \mathrm{H}, \mathrm{CH}_{2} \mathrm{NH}$ exchangeable with $\left.\mathrm{D}_{2} \mathrm{O}\right), 1.59$ (t, $\left.2 \mathrm{H}, \mathrm{NHCH}_{2} \mathrm{CH}=\right)$ (ppm) ; $\mathrm{MS}: \mathrm{M}^{+}$514. Anal.Calcd.for $\mathrm{C}_{28} \mathrm{H}_{26} \mathrm{~N}_{4} \mathrm{O}_{6}$; C, $65.36 ; \mathrm{H}, 5.05 ; \mathrm{N}, 10.89$. Found : C,65.33; H,5.07; $\mathrm{N}, 10.84$.

4 - (21-Thiobarbiturinyl) - 2 - (ochlorophenyl) - 3 - [(p- chlorophenylamino methylene)]- 2,3-dihydro-1,5-benzoxazepines (6g). $52 \%, \mathrm{mp} 200^{\circ} \mathrm{C}$ (ethanol); IR (KBr) $3329(\mathrm{NH}), 1690$,, 1710 (amidic $\mathrm{C}=\mathrm{O}$ ), 1630 (C-O-C), 1140 (C=S), cm ${ }^{1} ;{ }^{1} \mathrm{H} \mathrm{NMR}\left(\mathrm{CDCl}_{3}\right)$ d 9.36 (ss, 2H, 2NHCO), 3.62 (t, $1 \mathrm{H}, \mathrm{CH}-), 7.70-6.68(\mathrm{~m}, 12 \mathrm{H}, \mathrm{Ar}-\mathrm{H}) 3.75\left(\mathrm{~d}, 1 \mathrm{H}, \mathrm{C}_{3}{ }^{-}\right.$ $\mathrm{H}$ of thiazepine ring), $6.45\left(\mathrm{t}, 1 \mathrm{H}, \mathrm{C}_{2}-\mathrm{H}\right.$ of thiazepine ring), 3.05 (hump, $1 \mathrm{H}, \mathrm{CH}_{2} \mathrm{NH}$ exchangeable with $\left.\mathrm{D}_{2} \mathrm{O}\right), 1.58\left(\mathrm{t}, 2 \mathrm{H}, \mathrm{NHCH}_{2} \mathrm{CH}=\right)$ (ppm) ; $\mathrm{MS}: \mathrm{M}^{+} 538$. Anal.Calcd.for $\mathrm{C}_{26} \mathrm{H}_{19} \mathrm{~N}_{4} \mathrm{O}_{3} \mathrm{SCl}_{2} ; \mathrm{C}, 57.99 ; \mathrm{H}, 3.53$; $\mathrm{N}, 10.40$. Found: $\mathrm{C}, 57.97 ; \mathrm{H}, 3.56 ; \mathrm{N}, 10.38$.

4 - (21-Thiobarbiturinyl) - 2 - (ochlorophenyl) $-3-[(p-m e t h o x y p h e n y l a m i n o$ methylene)]-2,3-dihydrobenzoxazepines (6h). 69\%, $\mathrm{mp} 171^{\circ} \mathrm{C}$ (benzene); IR (KBr) $3345(\mathrm{NH}), 1700$, 1710 (amidic $\mathrm{C}=\mathrm{O}), 1625(\mathrm{C}=\mathrm{C}), 1440(\mathrm{C}=\mathrm{N}), 760$ (C-Cl), 1028 (C-O-C), $1148(\mathrm{C}=\mathrm{S}), 1230\left(\mathrm{OCH}_{3}\right) \mathrm{cm}$ ${ }^{1} ;{ }^{1} \mathrm{H} \mathrm{NMR}\left(\mathrm{CDCl}_{3}\right)$ d 9.30 (ss, 2H, 2NHCO), 3.68 (t, $1 \mathrm{H}, \mathrm{CH}-)$ 7.88-6.78 (m, 12H, Ar-H) $3.72\left(\mathrm{~d}, 1 \mathrm{H}, \mathrm{C}_{3}{ }^{-}\right.$ $\mathrm{H}$ of oxazepine ring), $6.59\left(\mathrm{t}, 1 \mathrm{H}, \mathrm{C}_{2}-\mathrm{H}\right.$ of oxazepine ring), 3.12 (hump, $1 \mathrm{H}, \mathrm{CH}_{2} \mathrm{NH}$ exchangeable with $\left.\mathrm{D}_{2} \mathrm{O}\right), 1.62\left(\mathrm{t}, 2 \mathrm{H}, \mathrm{NHCH}_{2} \mathrm{CH}=\right), 3.39\left(\mathrm{~s}, 3 \mathrm{H}, \mathrm{OCH}_{3}\right)$ (ppm) ; $\mathrm{MS}: \mathrm{M}^{+}$534.5. Anal.Calcd.for $\mathrm{C}_{27} \mathrm{H}_{23} \mathrm{~N}_{4} \mathrm{O}_{4} \mathrm{SCl}$; $\mathrm{C}, 60.61 ; \mathrm{H}, 4.30 ; \mathrm{N}, 10.47$. Found : $\mathrm{C}, 60.63 ; \mathrm{H}, 4.34$ ; N, 10.41 .

4 - (21-Thiobarbiturinyl) - 2 - (phydroxyphenyl) - 3 - [(p-chlorophenylamino methylene)]- 2,3-dihydro-1,5- benzoxazepines (6i). $48 \%, \mathrm{mp} 175^{\circ} \mathrm{C}$ (methanol); IR (KBr) $3347(\mathrm{NH})$, 1700, 1715, (amidic $\mathrm{C}=\mathrm{O}), 1630(\mathrm{C}=\mathrm{C}), 1460(\mathrm{C}=\mathrm{N})$, 730 (C-Cl), 1030 (C-O-C), 1145 (C=S), $3455(\mathrm{OH})$ $\mathrm{cm}^{-1} ;{ }^{1} \mathrm{H} \mathrm{NMR}\left(\mathrm{CDCl}_{3}\right)$ d 9.28 (ss, $2 \mathrm{H}, 2 \mathrm{NHCO}$ ), 3.60 (t, $1 \mathrm{H}, \mathrm{CH}-), 7.85-6.80(\mathrm{~m}, 12 \mathrm{H}, \mathrm{Ar}-\mathrm{H}), 3.77(\mathrm{~d}, 1 \mathrm{H}$, $\mathrm{C}_{3}-\mathrm{H}$ of oxazepine ring), $6.62\left(\mathrm{t}, 1 \mathrm{H}, \mathrm{C}_{2}-\mathrm{H}\right.$ of oxazepine ring), 3.15 (hump, $1 \mathrm{H}, \mathrm{CH}_{2} \mathrm{NH}$ exchangeable with $\mathrm{D}_{2} \mathrm{O}$ ), 1.58 (t, $2 \mathrm{H}, \mathrm{NHCH}_{2} \mathrm{CH}=$ ), $9.20(\mathrm{~s}, 1 \mathrm{H}, \mathrm{Ar}-\mathrm{OH})(\mathrm{ppm})$; MS : $\mathrm{M}^{+} 520.5$ Anal. Calcd.for $\mathrm{C}_{26} \mathrm{H}_{21} \mathrm{~N}_{4} \mathrm{O}_{4} \mathrm{SCl} ; \mathrm{C}, 59.94 ; \mathrm{H}, 4.03 ; \mathrm{N}, 10.75$. Found : $\mathrm{C}, 59.90 ; \mathrm{H}, 4.05 ; \mathrm{N}, 10.71$.

4 - (21-Thiobarbiturinyl) $-2-(p-$ hydroxyphenyl) $-3-[(p-m e t h o x y p h e n y l a m i n o$ methylene]-2,3- dihydro-1,5- benzoxazepines (6j). 
$65 \%$,mp 166C ${ }^{\circ}$ (methanal); IR(KBr) $3337(\mathrm{NH})$, $3460(\mathrm{OH}), 1680,1700$ (midic $\mathrm{C}=\mathrm{O}), 1640(\mathrm{C}=\mathrm{C})$, $1455(\mathrm{C}=\mathrm{N}), 1150$ (C=S), 1040(C-O-C) $\mathrm{Cm}^{1} ;{ }^{1} \mathrm{H}$ $\operatorname{NMR}\left(\mathrm{CDCl}_{3}\right), 7.80-6.68(\mathrm{~m}, 12 \mathrm{H}, \mathrm{Ar}-\mathrm{H}), 9.32(\mathrm{ss}, 2 \mathrm{H}$ $2 \mathrm{NHO}), 3.59(\mathrm{t}, 1 \mathrm{H}, \mathrm{CH}-), 3.75\left(\mathrm{~d}, 1 \mathrm{H}, \mathrm{C}_{3}-\mathrm{H}\right.$ of oxazepine ring), $3.45\left(\mathrm{~s}, 3 \mathrm{H}, \mathrm{OCH}_{3}\right), 9.15(\mathrm{~s}, 1 \mathrm{H}, \mathrm{Ar}-$ $\mathrm{OH}) 3.02$ (hump,) $1 \mathrm{H}, \mathrm{CH}_{2} \mathrm{NH}$ exchangeable with $\left.\mathrm{D}_{2} \mathrm{O}\right), 1.60\left(\mathrm{t}, 2 \mathrm{H} \mathrm{NHCH} \mathrm{CH}_{2}=\right), 6.40\left(\mathrm{t}, 1 \mathrm{H}, \mathrm{C}_{2}-\mathrm{H}\right.$ of oxazepine ring) (ppm); MS:+ $\mathrm{M}$ 516, Anal. calcd. fer $\mathrm{C}_{27} \mathrm{H}_{24} \mathrm{~N}_{4} \mathrm{O}_{5} \mathrm{~S} ; \mathrm{C}, 62.79 ; \mathrm{H}, 4.65 ; \mathrm{N}, 10.85$. Found: C, 62.74; H,4.62; N,10.87.

4 - (21-Thiobarbiturinyl) $-2-(\mathrm{m}-$

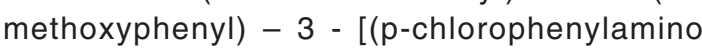
methylne)] - 2,3 - dihydro- 1-5 - benzoxazepines (6K). 50\%, mp $174^{\circ} \mathrm{C}$ (DMF/Water); IR (KBr) 3345 $(\mathrm{NH}), 1710,1715$ (amidic $\mathrm{C}=\mathrm{O}), 1133(\mathrm{C}=\mathrm{S}), 1615$ $(\mathrm{C}=\mathrm{C}), 1455(\mathrm{C}=\mathrm{N}), 720(\mathrm{C}-\mathrm{Cl}), 1040(\mathrm{C}-\mathrm{O}-\mathrm{C}) \mathrm{cm}-$ 1; ${ }^{1} \mathrm{H}$ NMR $\left(\mathrm{CDCl}_{3}\right) \delta 9.33$ (ss, $\left.2 \mathrm{H}, 2 \mathrm{NHCO}\right), 3.58$ $(\mathrm{t}, 1 \mathrm{H}, \mathrm{CH}-), 7.90-6.85(\mathrm{~m}, 12 \mathrm{H}, \mathrm{Ar}-\mathrm{H}), 3.73\left(\mathrm{~d}, 1 \mathrm{H}, \mathrm{C}_{3}-\right.$ $\mathrm{H}$ of oxazepine ring,), $6.65\left(\mathrm{t}, 1 \mathrm{H} \mathrm{C}_{2}-\mathrm{H}\right.$ of oxazepine ring), $3.34\left(\mathrm{~s}, 3 \mathrm{H}, \mathrm{OCH}_{3}\right), 3.20$ (hump, $1 \mathrm{H} \mathrm{CH} \mathrm{NH}_{2}$ exchangeable with $\left.\mathrm{D}_{2} \mathrm{O}\right), 1.60\left(\mathrm{t}, 2 \mathrm{H}, \mathrm{NH} \mathrm{CH}_{2} \mathrm{CH}=\right.$ ) (ppm;) MS; $\mathrm{M}^{+}$534.5.Anal Calcd.for $\mathrm{C}_{27} \mathrm{H}_{23} \mathrm{~N}_{4} \mathrm{O}_{4} \mathrm{SCl}$; C, 60.61; H, 4.30; N,1O.47. Found: C, 60.63; H, 4.27; $\mathrm{N}, 10.49$.

4-(21-Thiobarbiturinyl)-2(mmethoxyphenyl)-3[(p-methoxyphenylamino methylene)] -2,3-dihydro-1,5-benzoxazepines (6I). $45 \%, \mathrm{mp} 210^{\circ} \mathrm{C}$ (ethanol); IR ( $\left.\mathrm{KBr}\right) 3348(\mathrm{NH}), 1700$, 1710 (amidic $\mathrm{C}=\mathrm{O}), 1127$ ( $\mathrm{C}=\mathrm{S}), 1620(\mathrm{C}=\mathrm{C}), 1452$ $(\mathrm{C}=\mathrm{N}), \mathrm{t} 210\left(\mathrm{OCH}_{3}\right), 1048(\mathrm{C}-\mathrm{O}-\mathrm{C}) \mathrm{cm}^{-1} ;{ }^{1} \mathrm{H}$ NMR $\left(\mathrm{CDCl}_{3}\right) \delta 9.38$ (ss,2H, 2NHCO), $3.60(\mathrm{t}, 1 \mathrm{H}, \mathrm{CH}-)$, 7.88-6.74 (m,12H,Ar-H), $3.77\left(\mathrm{~d}, 1 \mathrm{H}, \mathrm{C}_{3}-\mathrm{H}\right.$ of oxazepine ring), $6.60\left(\mathrm{t}, 1 \mathrm{H}, \mathrm{C}_{2}-\mathrm{H}\right.$ of oxazepine ring), $3.40\left(\mathrm{~S}, 6 \mathrm{H}, 2 \times \mathrm{OCH}_{3}\right), 3.28$ (hump, $1 \mathrm{H}, \mathrm{CH}_{2} \mathrm{NH}$ exchangealele with $\left.\mathrm{D}_{2} \mathrm{O}\right), 1.62\left(\mathrm{t}, 2 \mathrm{H}, \mathrm{NHCH}_{2} \mathrm{CH}=\right)$ (ppm); MS: $\mathrm{M}^{+} 530$ Anal. Calcd.for $\mathrm{C}_{28} \mathrm{H}_{26} \mathrm{~N}_{4} \mathrm{O}_{5} \mathrm{~S}$; C, 63.39; H,4.90; N,1056. Found: C, 63.37; H, 4.43; N, 10.58 .

\section{Pharmacological evaluation}

Anticonvulsant activity. Maximum electroshock seizure (MES) test

This test was performed according to the method of Tomen et.al ${ }^{15}$. The group of ten rats was treated with test drugs $(50 \mathrm{mg} / \mathrm{kg}$ i.p.) phenytoin sodium (30mg/kg i.p.). After $1 \mathrm{~h}$, they were subjected to the shock of $150 \mathrm{~mA}$ by convulsiometer through ear electrodes for $0.2 \mathrm{~s}$ and the presence or absence of extensor response was noted. Animals in which extensor response was abolished were taken as protected rats.

Pentylenetetrazole (PTZ) induced seizures test. This test was performed by following the method of Fischer ${ }^{16}$. The rats were injected with pentylenetetrazol in dose of $70 \mathrm{mg} / \mathrm{kg}$ subcutaneously in scruff of neck. After 2-4 min of PTZ injection animals developed sequence of excitement, myoclonic jerks, clonic seizures, one or more maximum tonic seizures. Animals exhibiting these seizures patterns were selected standard drug used in this model was sodium valproate (80 $\mathrm{mg} / \mathrm{kg}$ i.p) and was injected $60 \mathrm{~min}$ prior to PTZ challenge.

\section{Approximate lethal dose (ALD $\left.D_{50}\right)$}

Approximate $50 \%$ lethal dose $\left(A L D_{50}\right)$ of the compounds were determined in albino mice. The mice of either sex 20-25g were used. The test compounds were injected intraperitoneally at different dose levels in groups of 10 animals. After $24 \mathrm{~h}$ of drug administration, percent mortality in each group was abserved from the data obtained. ALD $_{50}$ was calculated by the method of Smith ${ }^{17}$.

\section{CONCLUSION}

While considering all the newly synthesized compounds of this series together, we may conclude that:

1. 2-Thiobarbituric acid, containing compounds were found to possess potent anticonvulsant activity in comparison to 2oxobarbituric acid, containing compounds.

2. Presence of benzothiazepine moiety has shown better anticonvulsant activity than the compounds having benzoxazepine moiety.

3. Compounds having benzothiazepine moiety with thiobarbituric acid showed better anticonvulsant activity than the compounds having benzoxazepine moiety with oxobarbituric acid.

4. 2-Chlorophenyl substitution at second position of benzothiazepines ring showed more potent activity than other substituted benzothiazepines.

5. Presence of electronegative atom (chlorine) 
plays a pivotal role to increase the anticonvulsant activity. Regarding acute toxicity studies it may be concluded that all the compounds showed high value of ALD ${ }_{50}$ thus indicating a good safety margin.

\section{ACKNOWLEDGEMENTS}

The authors are thankful to Central Drug Research Institute (CDR1), Lucknow, India for elemental and spectral analysis. One of us (Dr. MirdulaTyagi) is thankful to University Grants Comission (UGC) New Delhi, for the award of PDF.

\section{REFERENCES}

1. Goodman and Gilman's The Pharmacological Basis of Therapeutics ; McGraw-Hill : New York, 1996; p 471.

2. Goodman and Gilman's The Pharmacological Basis of Therapeutics ; McGraw-Hill : New York, 1996; p 472.

3. Siddiqui N.A. \&Ahsan W., Arch PharmaChem, Like Sci, 2009, 342, 173.

4. Goel B., Sharma S., Bajaj K., Bansal E., Singh T., Malik N., Lata S., Tygai C., Panwar H., Agarwal A. \& Kumar A., Indian J. Pharma. Sci, 2005, 67194.

5. Archana, Srivastav V.K. \& Kumar A., Bioorganic and Med. Chem. (2004) 1257.

6. Archana, Rani P., Bajaj K., Srivastava V.K., Chandra R. \& Kumar A., Arzneim. Forsch / Drug Res. 53 (2003) 301.

7. Sarma G.V.S.P., Rao J.V. \& Suresh B., Chem. Abstr, 2000, 133, $120291 \mathrm{~g}$.

8. Osman A. N., Kandel M.M. \& Ahmed M.,
Indian . J. Chem. ,1996., 35B 1073.

9. Garg N., Chandra T., Archana, Jain B.A. \& Kumar A., Eur. J. Med. Chem., 2010, 451529.

10. Zhong T.P., Guan L.P., Li-Mingzhao, Hu-PiPio\& Shan Zuan, Eur, J. Med. Chem. 2008 ., 431216.

11. Bajaj K., Archana\& Kumar A., Eur. J. Med. Chem. 2004.,39 ,369.

12. Bajaj K., Srivastave V.K. \& Kumar A., Indian. J. Chem. 2003., 42 B 1149.

13. Youssef, K.M. \& Said M.M., Egypt. J. Pharm. Sci. 1996., $37,45$.

14. Sarro G.D., Chimmirri A., Sarro A.D., Gittu R., Grasso S. \&Zappala M., Eur. J. Med. Chem. 1995., 30, 925.

15. Toman J.E.P. Swingarel E.A. \&Goudman L.S., Neuro J. Physiol (1946) 231.

16. Fisher R.S. Brain Res. Rev. ; 1989., 14; 245.

17. Smith Q.E., J. Pharmacol. Exp. Ther. 1950, 100408. 